\title{
Synthesis and Catalytic Reactivity of Cobalt Pincer Nitrosyl Hydride Complexes
}

Jan Pecak, ${ }^{\dagger}$ Sarah Fleissner, ${ }^{\dagger}$ Luis F. Veiros, ${ }^{\S}$ Ernst Pittenauer, Karl Kirchner ${ }^{\dagger, *}$

† Institute of Applied Synthetic Chemistry, Vienna University of Technology, Getreidemarkt 9, A-1060 Vienna, AUSTRIA.

${ }^{\S}$ Centro de Química Estrutural and Departamento de Engenharia Química, Instituto Superior Técnico, Universidade de Lisboa, Av Rovisco Pais, 1049-001 Lisboa, PORTUGAL.

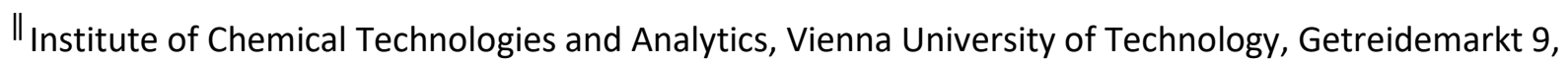
A-1060 Vienna, AUSTRIA.

${ }^{\ddagger}$ X-Ray Center, Vienna University of Technology, Getreidemarkt 9, A-1060 Vienna, AUSTRIA

Supporting Information 

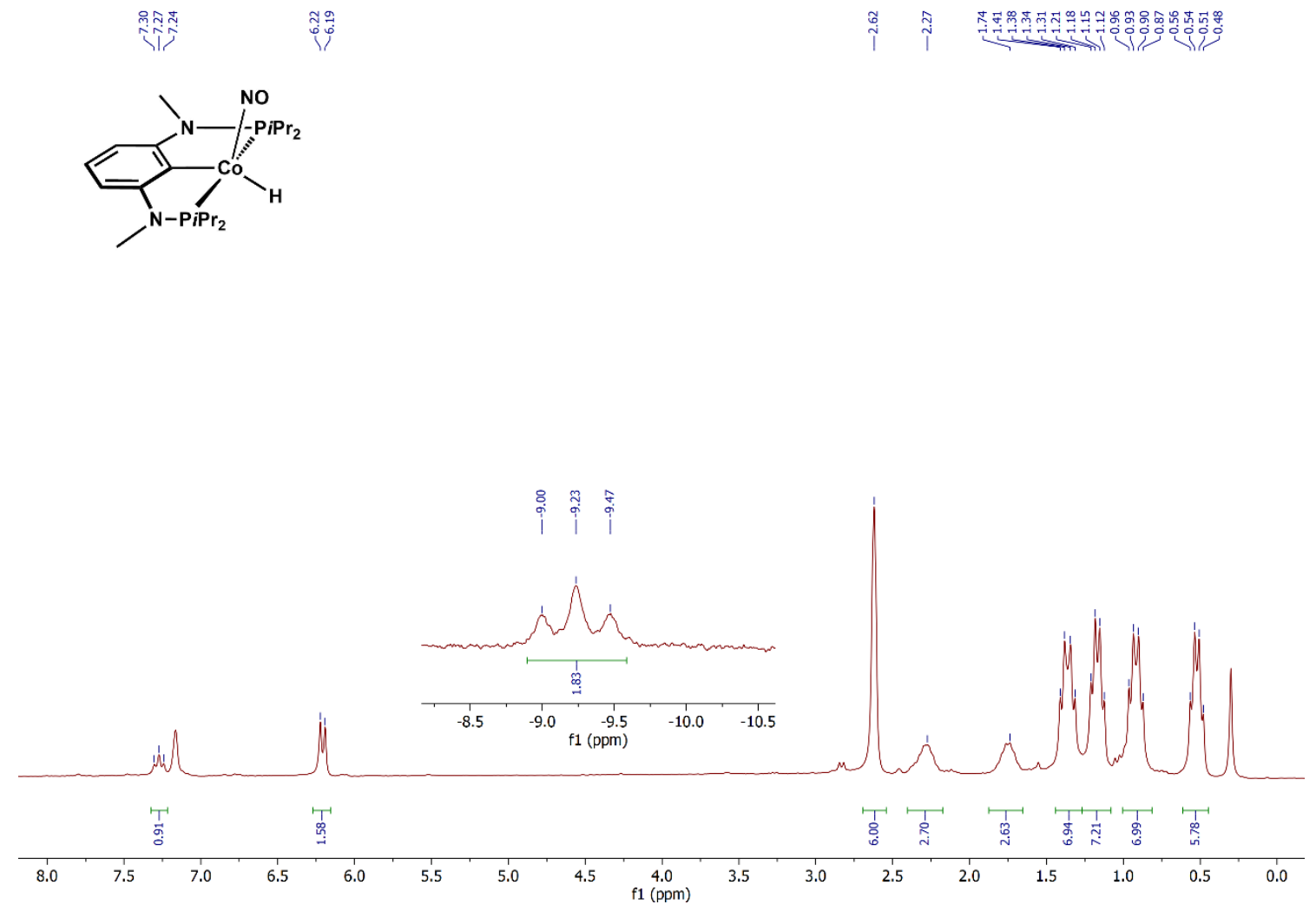

Figure S1. ${ }^{1} \mathrm{H}$ NMR Spectrum of $\left[\mathrm{Co}\left(\mathrm{PCPNMe}_{-}-\mathrm{Pr}\right)(\mathrm{NO})(\mathrm{H})\right](2 \mathrm{a})$.

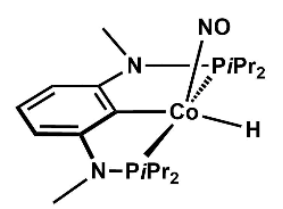

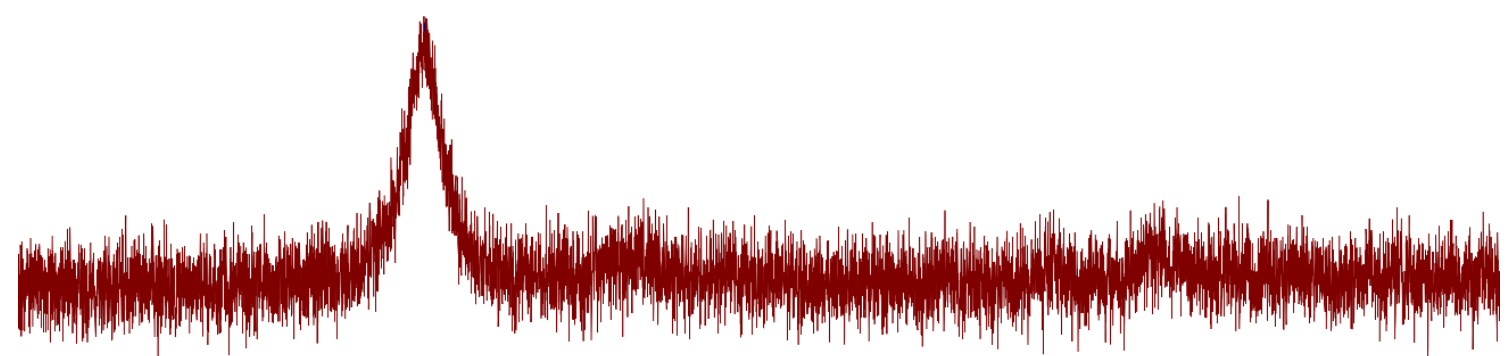
$\begin{array}{lllllllllllllllllllllllllllll}1 & 1 \\ 240 & 230 & 220 & 210 & 200 & 190 & 180 & 170 & 160 & 150 & 140 & 130 & 120 & 110 & 100 & 90 & 80 & 70 & 60 & 50 & 40 & 30 & 20 & 10 & 0\end{array}$

Figure S2. ${ }^{31} \mathrm{P}\left\{{ }^{1} \mathrm{H}\right\}$ NMR Spectrum of $\left[\mathrm{Co}\left(\mathrm{PCP}^{\mathrm{NM}}-\mathrm{PPr}\right)(\mathrm{NO})(\mathrm{H})\right](\mathbf{2 a})$. 


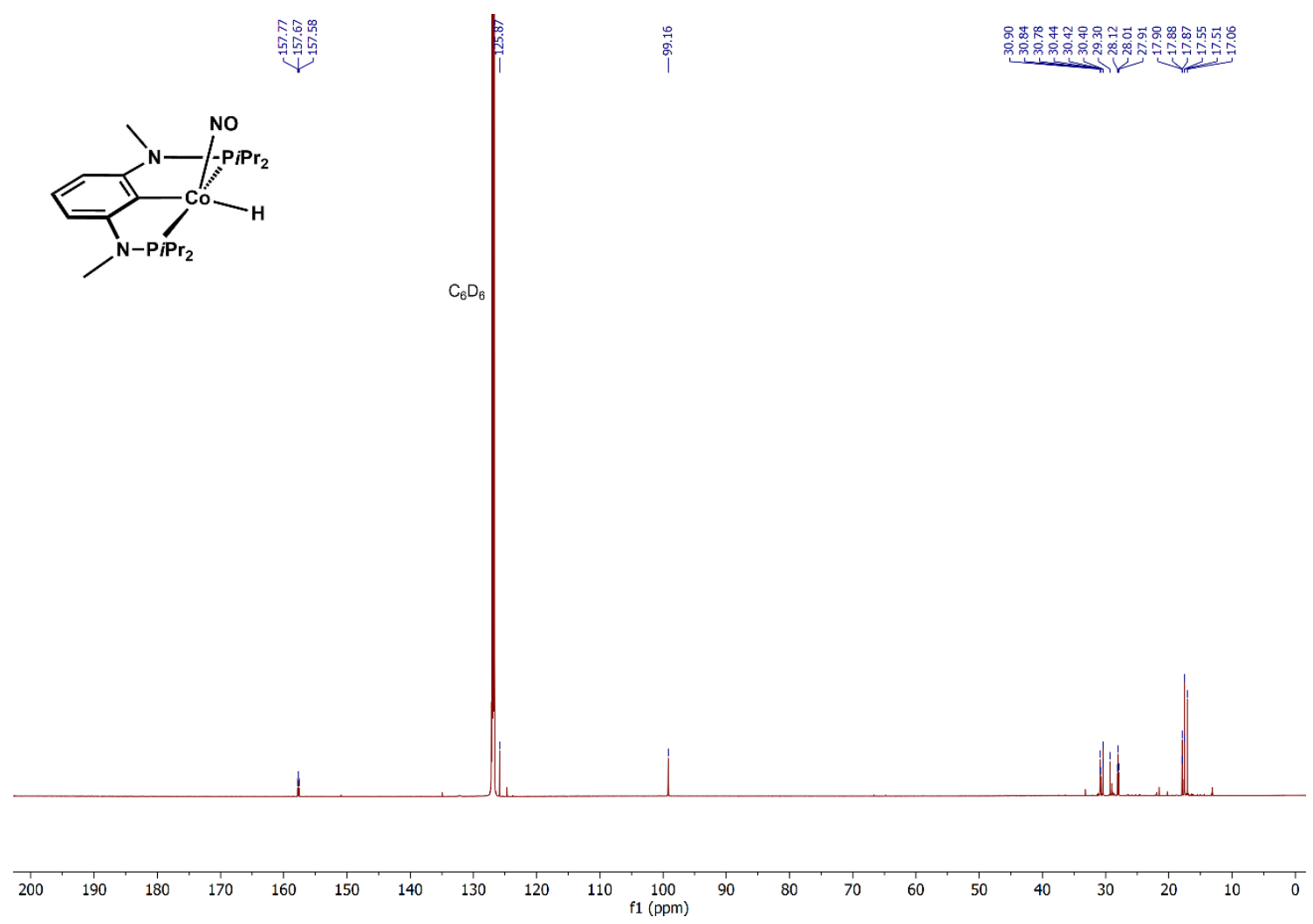

Figure S3. ${ }^{13} \mathrm{C}$ NMR Spectrum of $\left[\mathrm{Co}\left(\mathrm{PCP}^{\mathrm{NMe}}-\mathrm{Pr}\right)(\mathrm{NO})(\mathrm{H})\right](\mathbf{2 a})$.
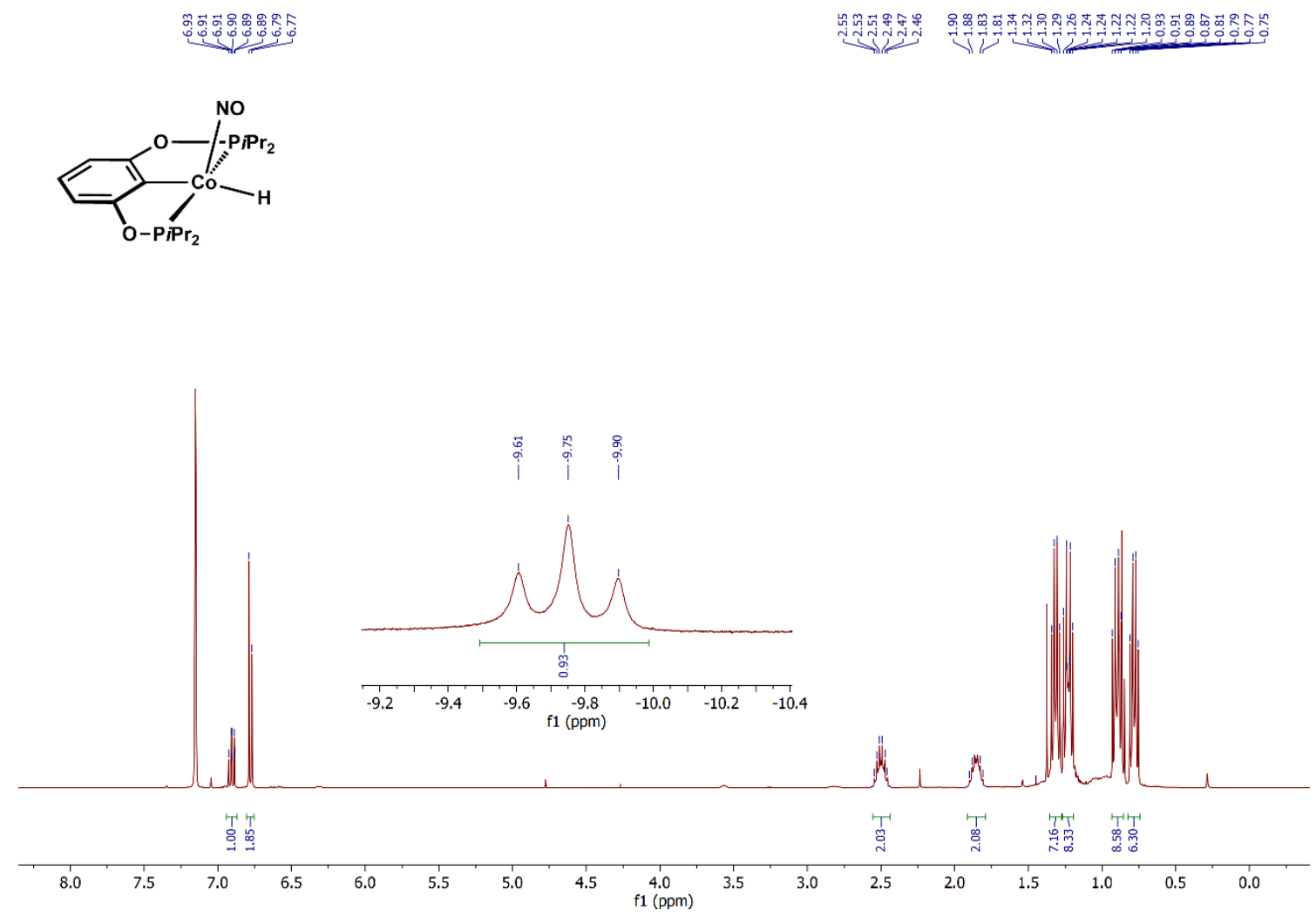

Figure S4. ${ }^{1} \mathrm{H}$ NMR Spectrum of $\left[\mathrm{Co}\left(\mathrm{PCP}^{\mathrm{O}}-\mathrm{iPr}\right)(\mathrm{NO})(\mathrm{H})\right](\mathbf{2 b})$. 
$\stackrel{\overrightarrow{0}}{\vec{i}}$
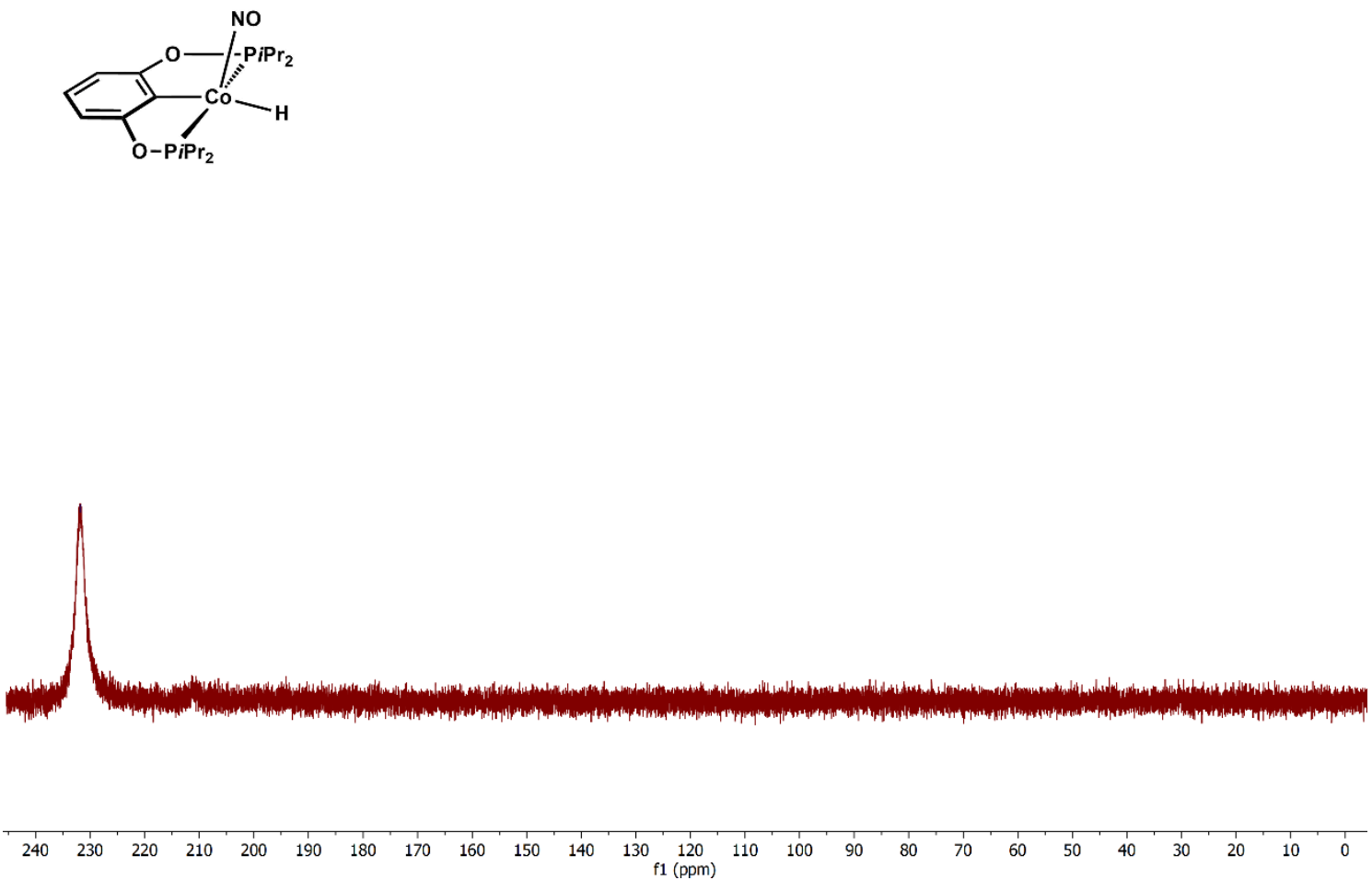

Figure S5. ${ }^{31} \mathrm{P}\left\{{ }^{1} \mathrm{H}\right\}$ NMR Spectrum of $\left[\mathrm{Co}\left(\mathrm{PCP}^{\mathrm{O}}-\mathrm{PPr}\right)(\mathrm{NO})(\mathrm{H})\right](\mathbf{2 b})$.

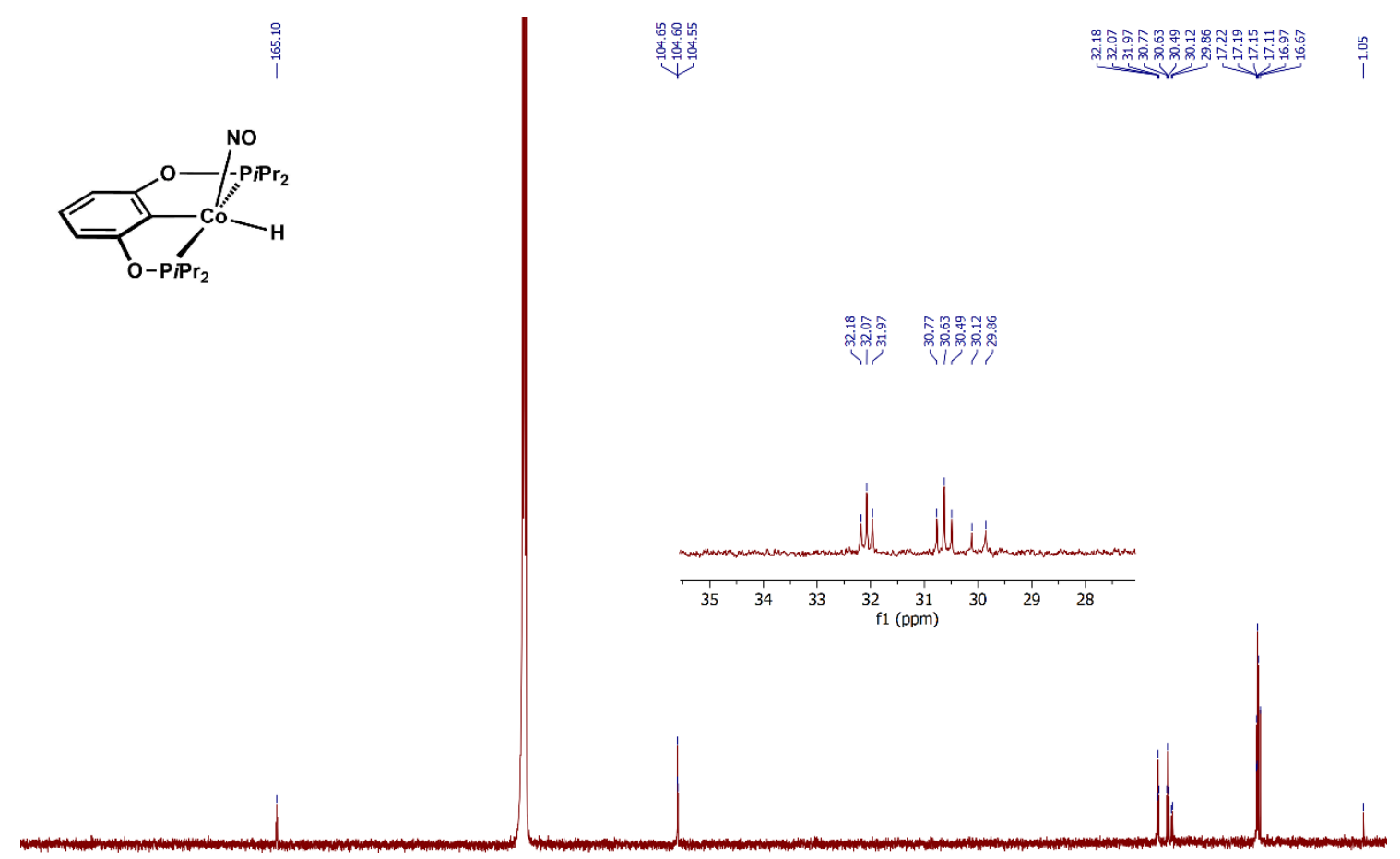

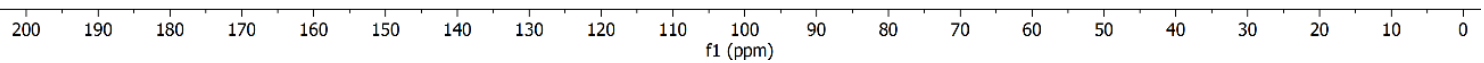

Figure S6. ${ }^{13} \mathrm{C}$ NMR Spectrum of $\left[\mathrm{Co}\left(\mathrm{PCP}^{\mathrm{O}}-\mathrm{PPr}\right)(\mathrm{NO})(\mathrm{H})\right](\mathbf{2 b})$. 


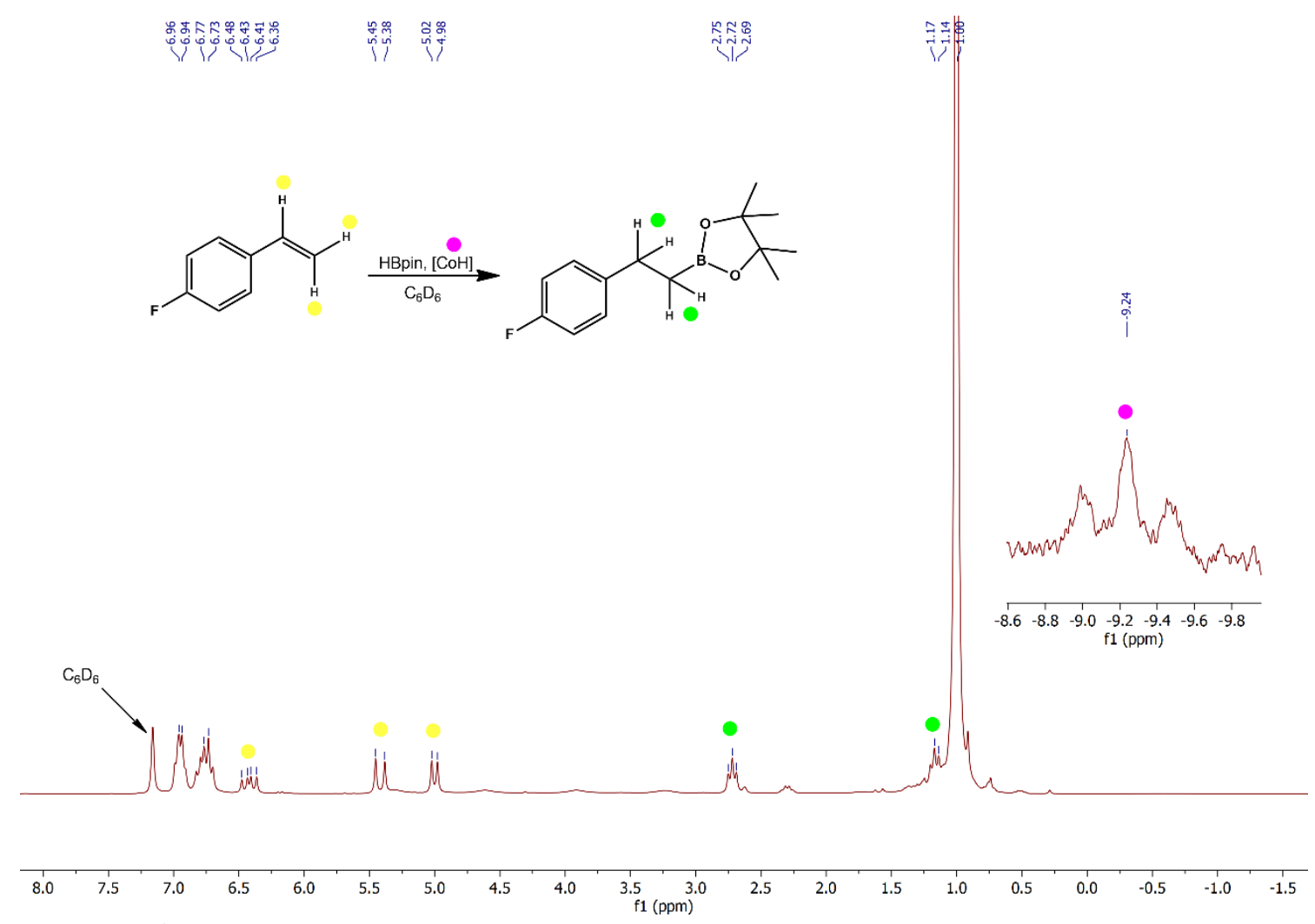

Figure S7. ${ }^{1} \mathrm{H}$ NMR monitoring of the catalytic transformation of 4 -fluorostyrene with $\mathrm{HBpin}$ and $2 \mathrm{~mol} \%$ of $2 \mathrm{a}$ in benzene- $\mathrm{d}_{6}$ solution after $3 \mathrm{~h}$ at $25^{\circ} \mathrm{C}$. Reaction vial and NMR were prepared in a Glovebox.
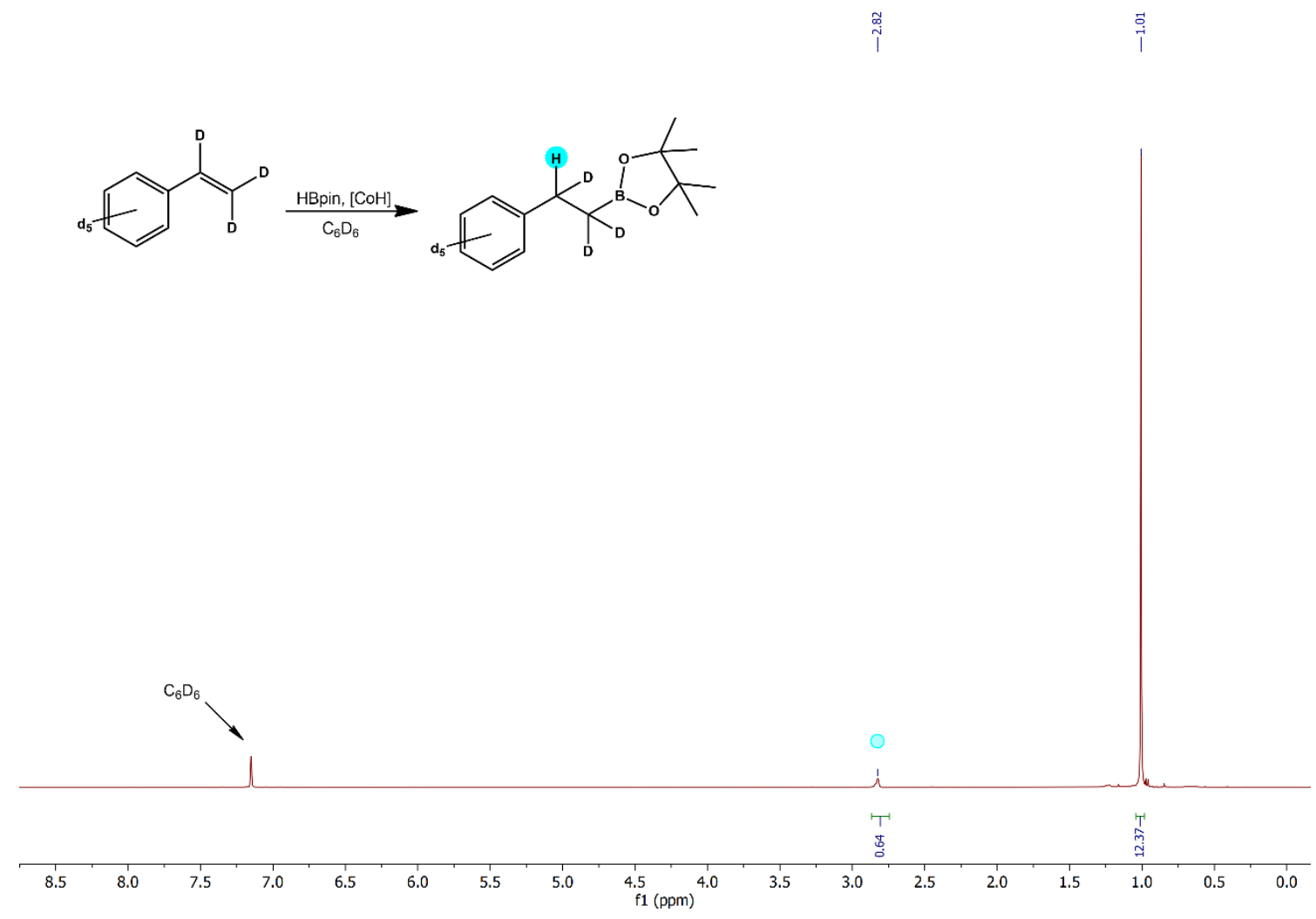

Figure S8. ${ }^{1} \mathrm{H}$ NMR spectrum of deuterium labelling experiment using styrene- $\mathrm{d}_{8}$ as substrate and ${ }^{1} \mathrm{HBpin}$ with $1 \mathrm{a}$ as precatalyst in benzene- $\mathrm{d}_{6}$ solution after $24 \mathrm{~h}$. 
SG

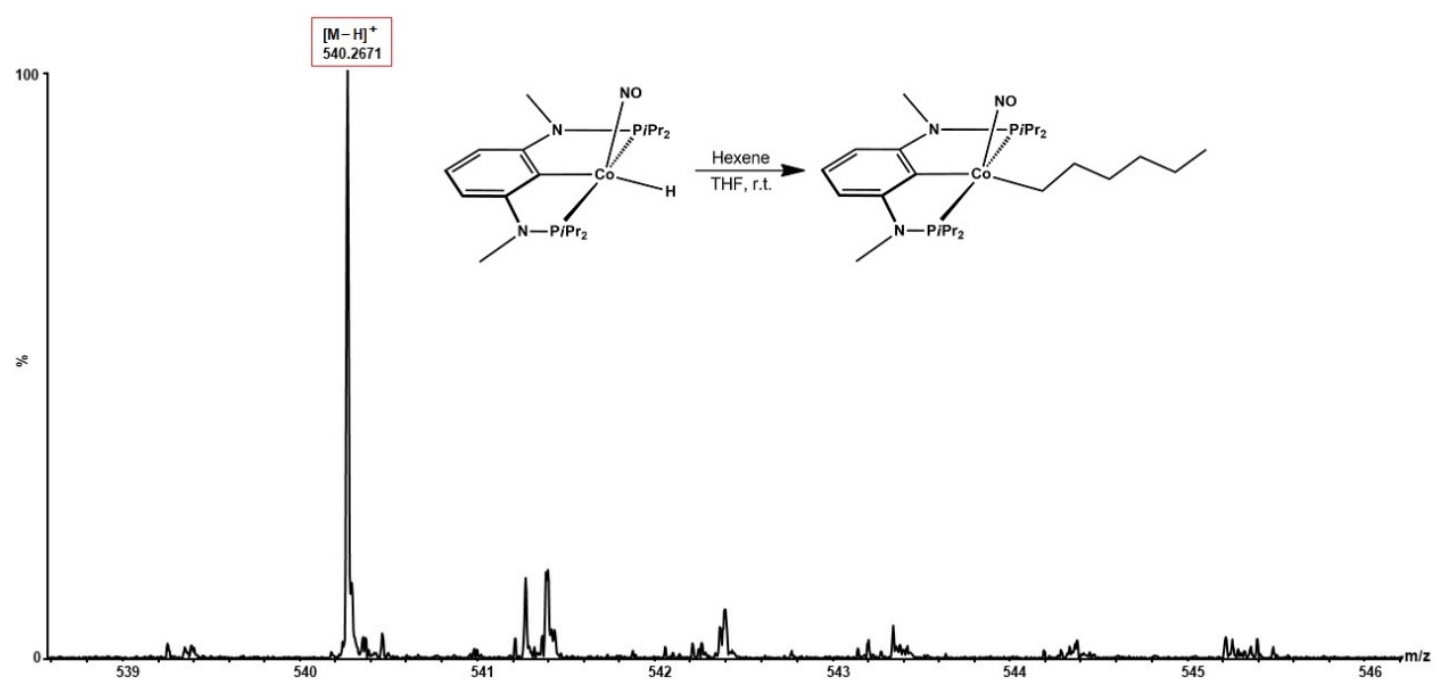

Figure S9. Reaction monitoring using high-resolution ESI-MS and direct infusion technique. 1-Hexene was used as a substrate with $\mathbf{2 a}$ and HBpin in THF; measurement after $1 \mathrm{~h}$. Mass error $=1.2 \mathrm{ppm}$.

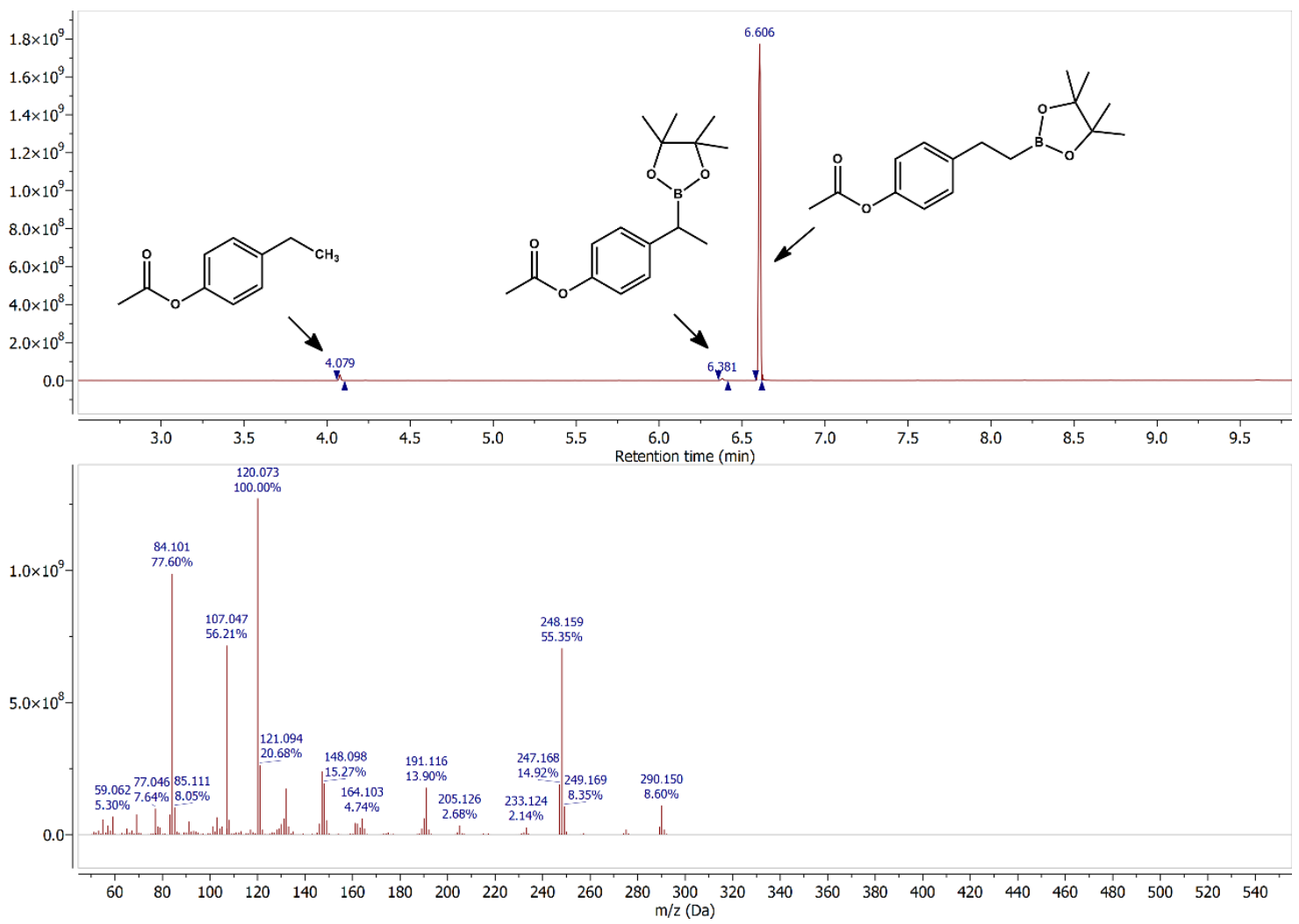

Figure S10. Exemplary chromatogram of catalytic transformation of 4-vinylphenyl acetate as substrate with HBpin and $1 \mathrm{a}$ after $24 \mathrm{~h}$ in benzene. MS spectrum below shows the main product (290 D). 

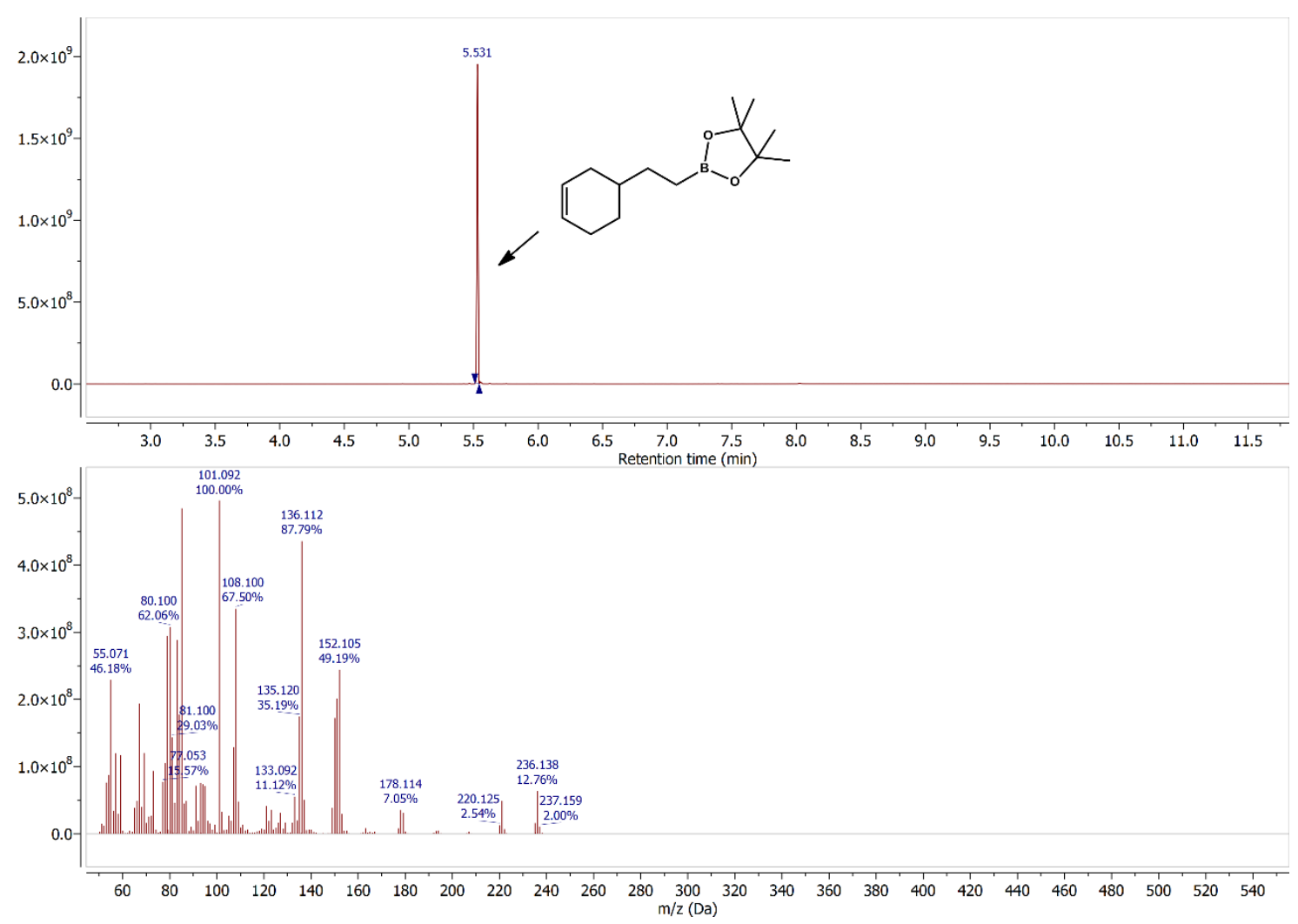

Figure S11. Chromatogram of catalytic transformation of 4-Vinyl-1-cyclohexene with HBpin and 1a in benzene. MS spectrum below shows the single product with isotope pattern (236 D).

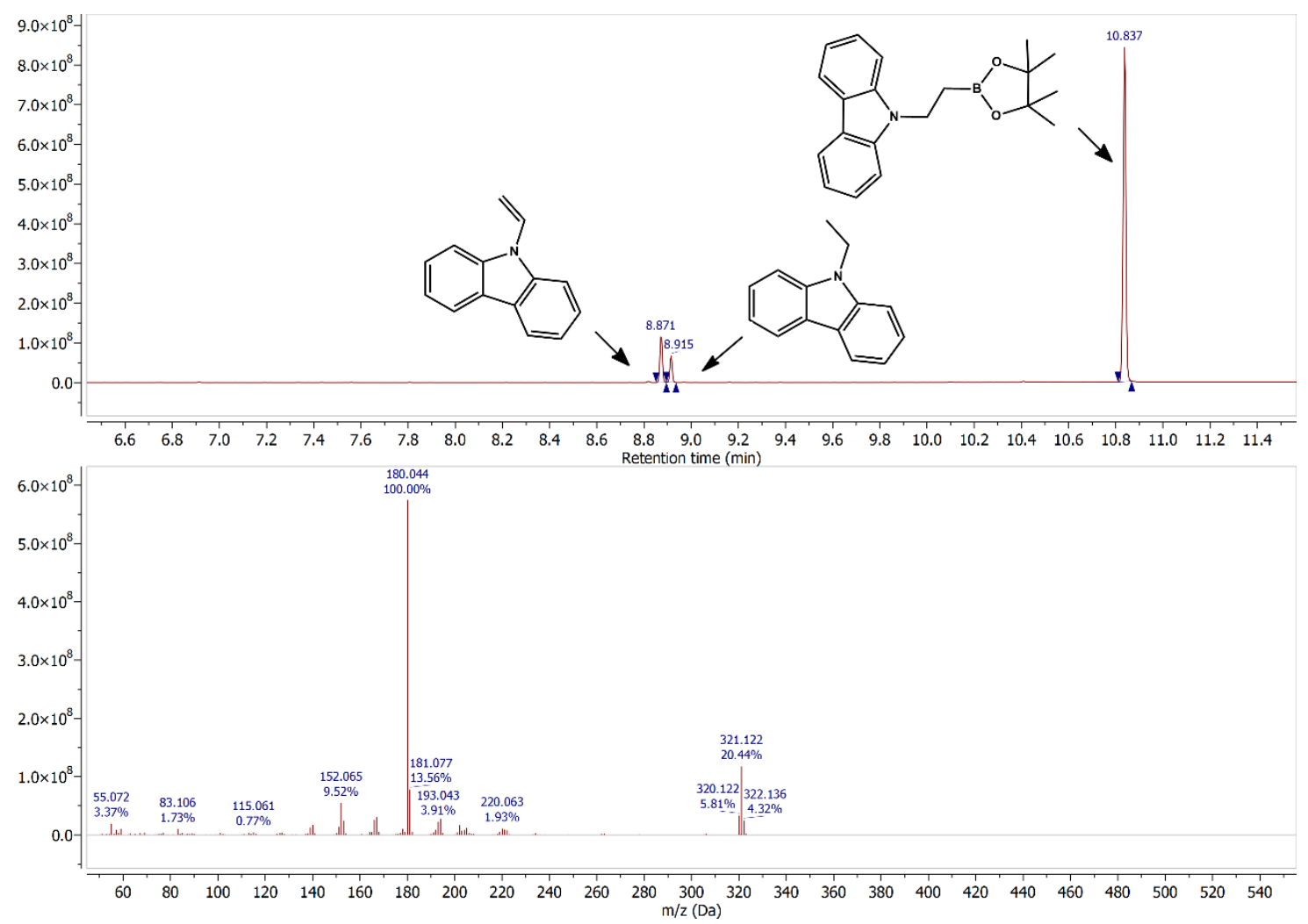

Figure S12. Chromatogram of catalytic transformation of N-Vinylcarbazole with HBpin and 1a in benzene. MS spectrum below shows the single product with isotope pattern (321 D). 

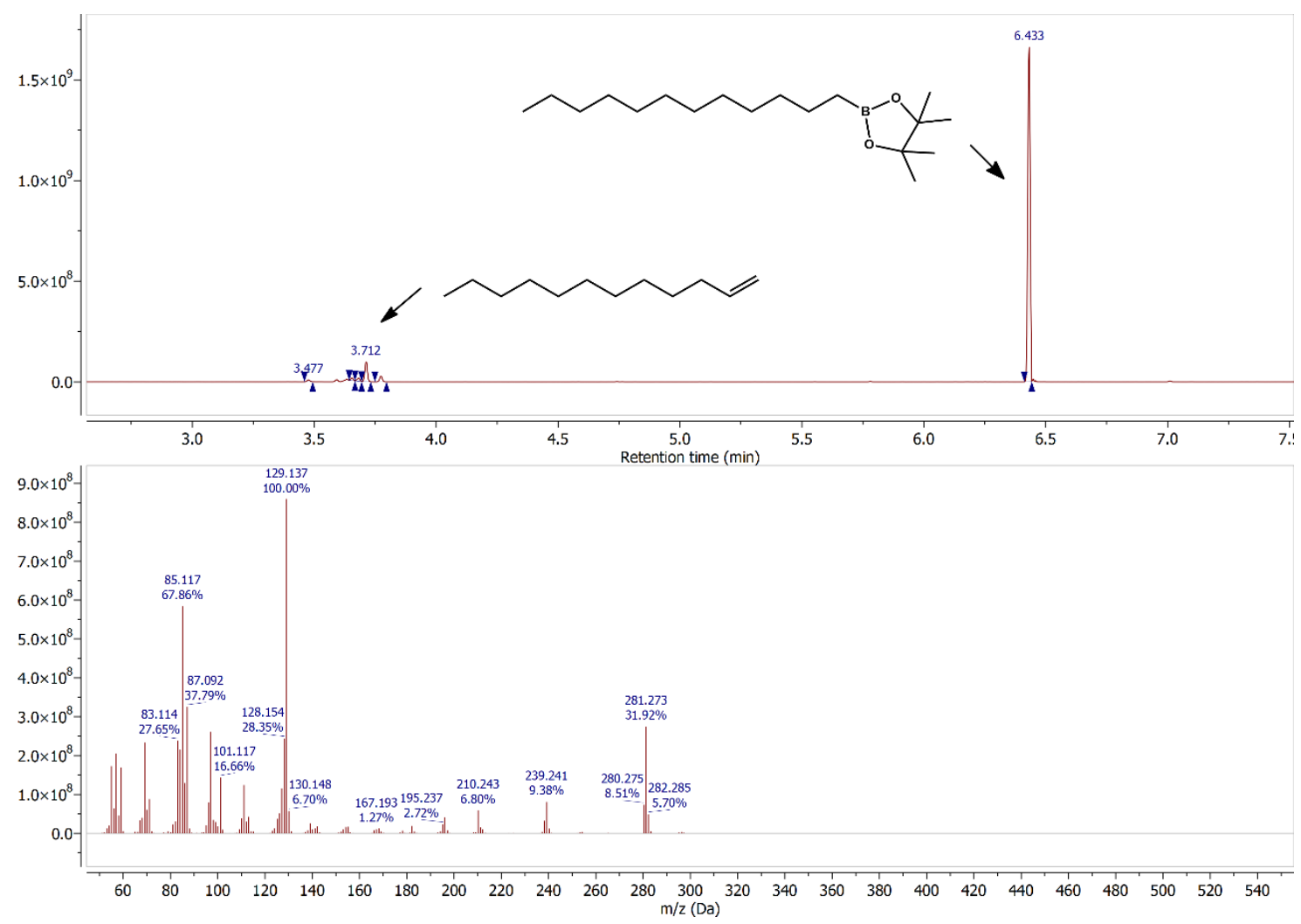

Figure S13. Chromatogram of catalytic transformation of 1-dodecene with $\mathrm{HBpin}$ and $1 \mathrm{a}$ in benzene. MS spectrum below shows the single product with isotope pattern (281 D, $\mathrm{M}-\mathrm{CH}_{2}$ ).
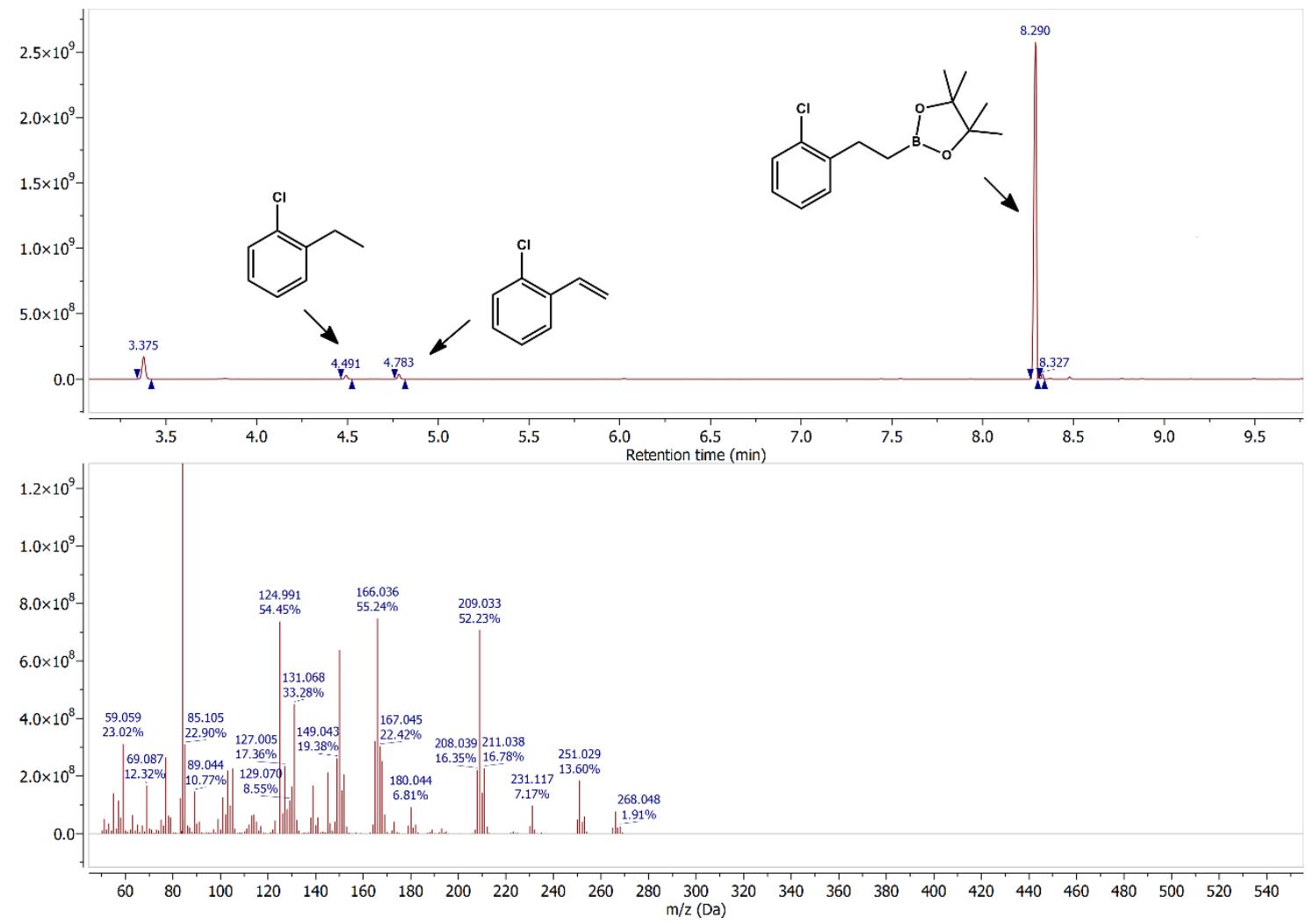

Figure S14. Chromatogram of catalytic transformation of 2-chlorostyrene with HBpin and 3a in benzene. MS spectrum below shows the single product with isotope pattern (266 D). 


\section{NMR Spectra of organic products}

2-(4-fluorophenethyl)-4,4,5,5-tetramethyl-1,3,2-dioxaborolane: ${ }^{[1]}$ colorless oil, ${ }^{1} \mathrm{H} N M R(250 \mathrm{MHz}, \delta$, $\mathrm{CD}_{2} \mathrm{Cl}_{2}$ ): $7.22(\mathrm{dd}, J=8.8,5.5 \mathrm{~Hz}, 2 \mathrm{H}), 6.98(\mathrm{t}, J=8.9 \mathrm{~Hz}, 2 \mathrm{H}), 2.73(\mathrm{t}, J=8.0 \mathrm{~Hz}, 2 \mathrm{H}), 1.23(\mathrm{~s}, 12 \mathrm{H}), 1.10$ $(\mathrm{t}, J=7.9 \mathrm{~Hz}, 2 \mathrm{H}) \cdot{ }^{13} \mathrm{C}\left\{{ }^{1} \mathrm{H}\right\} \mathrm{NMR}\left(63 \mathrm{MHz}, \delta, \mathrm{CD}_{2} \mathrm{Cl}_{2}\right): 161.1(\mathrm{~d}, J=241.8 \mathrm{~Hz}), 129.4(\mathrm{~d}, J=7.8 \mathrm{~Hz}), 114.6$ $(\mathrm{d}, \mathrm{J}=21.0 \mathrm{~Hz}), 83.0,29.1,24.6 .{ }^{19} \mathrm{~F} \mathrm{NMR}\left(235 \mathrm{MHz}, \delta, \mathrm{CD}_{2} \mathrm{Cl}_{2}\right):-119.1$.

4,4,5,5-tetramethyl-2-(4-methylphenethyl)-1,3,2-dioxaborolane: ${ }^{[2]}$ colorless oil, ${ }^{1} \mathrm{H}$ NMR $(250 \mathrm{MHz}, \delta$, $\left.\mathrm{CD}_{2} \mathrm{Cl}_{2}\right): 7.12(\mathrm{~m}, 4 \mathrm{H}), 2.71(\mathrm{t}, J=9.3 \mathrm{~Hz}, 2 \mathrm{H}), 2.34(\mathrm{~s}, 3 \mathrm{H}), 1.25(\mathrm{~s}, 12 \mathrm{H}), 1.10(\mathrm{t}, J=8.0 \mathrm{~Hz}, 2 \mathrm{H}) .{ }^{13} \mathrm{C}\left\{{ }^{1} \mathrm{H}\right\}$ $\operatorname{NMR}\left(63 \mathrm{MHz}, \delta, \mathrm{CD}_{2} \mathrm{Cl}_{2}\right): 141.5,134.9,128.7,127.8,82.9,29.5,24.6,20.6$.

4-(2-(4,4,5,5-tetramethyl-1,2,3-dioxaborolan-2-yl)ethyl)phenyl acetate: ${ }^{[2]}$ colorless oil, ${ }^{1} \mathrm{H}$ NMR (250 $\left.\mathrm{MHz}, \delta, \mathrm{CD}_{2} \mathrm{Cl}_{2}\right): 7.25(\mathrm{~d}, J=8.2 \mathrm{~Hz}, 2 \mathrm{H}), 6.99(\mathrm{~d}, J=8.5 \mathrm{~Hz}, 2 \mathrm{H}), 2.75(\mathrm{t}, J=8.2 \mathrm{~Hz}, 2 \mathrm{H}), 2.28(\mathrm{~s}, 3 \mathrm{H})$, $1.24(\mathrm{~s}, 12 \mathrm{H}), 0.89(\mathrm{t}, J=7.2 \mathrm{~Hz}, 2 \mathrm{H}) \cdot{ }^{13} \mathrm{C}\left\{{ }^{1} \mathrm{H}\right\} \mathrm{NMR}\left(63 \mathrm{MHz}, \delta, \mathrm{CD}_{2} \mathrm{Cl}_{2}\right): 142.2,128.8,121.2,83.0,29.3$, 24.6, 20.8 .

2-(4-chlorophenethyl)-4,4,5,5-tetramethyl-1,3,2-dioxaborolane: ${ }^{[2]}$ colorless oil, ${ }^{1} \mathrm{H} \mathrm{NMR}(250 \mathrm{MHz}, \delta$, $\left.\mathrm{CD}_{2} \mathrm{Cl}_{2}\right): 7.35-7.14(\mathrm{~m}, 4 \mathrm{H}), 2.73(\mathrm{t}, J=8.4 \mathrm{~Hz}, 2 \mathrm{H}), 1.23(\mathrm{~s}, 12 \mathrm{H}), 1.10(\mathrm{t}, J=7.7 \mathrm{~Hz}, 2 \mathrm{H}) .{ }^{13} \mathrm{C}\left\{{ }^{1} \mathrm{H}\right\} \mathrm{NMR}$ $\left(63 \mathrm{MHz}, \delta, \mathrm{CD}_{2} \mathrm{Cl}_{2}\right.$ ): 143.2, 130.9, 129.4, 128.1, 83.1, 29.3, 24.6.

4,4,5,5,-tetramethyl-2-phenethyl-1,3,2-dioxaborolane: ${ }^{[2]}$ colorless oil, ${ }^{1} \mathrm{H} N M R\left(250 \mathrm{MHz}, \delta, \mathrm{CDCl}_{3}\right)$ : 7.42-7.12 (m, 5H), $2.80(\mathrm{t}, J=8.3 \mathrm{~Hz}, 2 \mathrm{H}), 1.26(\mathrm{~s}, 12 \mathrm{H}), 1.20(\mathrm{t}, J=8.3 \mathrm{~Hz}, 2 \mathrm{H}) .{ }^{13} \mathrm{C}\left\{{ }^{1} \mathrm{H}\right\} \mathrm{NMR}(63 \mathrm{MHz}$, $\left.\delta, \mathrm{CD}_{2} \mathrm{Cl}_{2}\right): 144.4,128.2,128.0,125.5,83.1,29.9,24.8$.

2-hexyl-4,4,5,5-tetramethyl-1,3,2-dioxaborolane: ${ }^{[3]}$ colorless oil, ${ }^{1} \mathrm{H}$ NMR $\left(400 \mathrm{MHz}, \delta, \mathrm{CD}_{2} \mathrm{Cl}_{2}\right): 1.32-$ $1.15(\mathrm{~m}, 8 \mathrm{H}), 1.13(\mathrm{~s}, 12 \mathrm{H}), 0.79(\mathrm{t}, J=7.0 \mathrm{~Hz}, 3 \mathrm{H}), 0.63(\mathrm{t}, J=7.6 \mathrm{~Hz}, 2 \mathrm{H}) .{ }^{13} \mathrm{C}\left\{{ }^{1} \mathrm{H}\right\} \mathrm{NMR}(101 \mathrm{MHz}, \delta$, $\left.\mathrm{CD}_{2} \mathrm{Cl}_{2}\right): 86.7,32.0,31.6,24.6,24.0,22.6,13.8$.

9-(2-(4,4,5,5-tetramethyl-1,3,2-dioxaborolan-2-yl)ethyl)-9H-carbazole: ${ }^{[2]}$ colorless oil, ${ }^{1} \mathrm{H}$ NMR (250 $\left.\mathrm{MHz}, \delta, \mathrm{CDCl}_{3}\right): 8.06(\mathrm{~d}, J=7.7 \mathrm{~Hz}, 1 \mathrm{H}), 7.53-7.31(\mathrm{~m}, 4 \mathrm{H}), 7.29-7.11(\mathrm{~m}, 2 \mathrm{H}), 4.43(\mathrm{t}, J=7.4 \mathrm{~Hz}, 2 \mathrm{H})$, 1.46-1.35 (m, 2H), $1.18(\mathrm{~s}, 12 \mathrm{H}) .{ }^{13} \mathrm{C}\left\{{ }^{1} \mathrm{H}\right\} \mathrm{NMR}\left(63 \mathrm{MHz}, \delta, \mathrm{CDCl}_{3}\right): 125.5,120.3,118.7,109.1,83.6$, $38.8,24.9$.

Trimethyl(3-(4,4,5,5-tetramethyl-1,3,2-dioxaborolan-2-yl)propyl)silane: ${ }^{[2]}$ colorless oil, ${ }^{1} \mathrm{H}$ NMR (400 $\left.\mathrm{MHz}, \delta, \mathrm{CD}_{2} \mathrm{Cl}_{2}\right): 1.48-1.39(\mathrm{~m}, 2 \mathrm{H}), 1.24(\mathrm{~s}, 12 \mathrm{H}), 0.80(\mathrm{t}, J=7.6 \mathrm{~Hz}, 2 \mathrm{H}), 0.58-0.52(\mathrm{~m}, 2 \mathrm{H}), 0.00(\mathrm{~s}$, 9H). ${ }^{13} \mathrm{C}\left\{{ }^{1} \mathrm{H}\right\}$ NMR $\left(101 \mathrm{MHz}, \delta, \mathrm{CD}_{2} \mathrm{Cl}_{2}\right): 82.7,30.0,24.6,19.9,18.5,-2.0$.

4,4,5,5-tetramethyl-2-(3-phenylpropyl)-1,3,2-dioxaborolane: ${ }^{[2]}$ colorless oil, ${ }^{1} \mathrm{H}$ NMR $(250 \mathrm{MHz}, \delta$, $\left.\mathrm{CD}_{2} \mathrm{Cl}_{2}\right)$ : 7.38-7.15 (m, 5H), $2.65(\mathrm{t}, J=8.0 \mathrm{~Hz}, 2 \mathrm{H}), 1.74(\mathrm{p}, J=7.7 \mathrm{~Hz}, 2 \mathrm{H}), 1.27(\mathrm{~s}, 12 \mathrm{H}), 0.82(\mathrm{t}, J=7.8$ $\mathrm{Hz}, 2 \mathrm{H}) .{ }^{13} \mathrm{C}\left\{{ }^{1} \mathrm{H}\right\} \mathrm{NMR}\left(63 \mathrm{MHz}, \delta, \mathrm{CD}_{2} \mathrm{Cl}_{2}\right): 142.9,128.5,128.1,125.5,82.8,38.4,26.2,24.6$.

2-(2-cyclohexylethyl)-4,4,5,5-tetramethyl-1,3,2-dioxaborolane: ${ }^{[2]}$ colorless oil, ${ }^{1} \mathrm{H} \mathrm{NMR}(250 \mathrm{MHz}, \delta$, $\left.\mathrm{CDCl}_{3}\right): 1.76-1.56(\mathrm{~m}, 5 \mathrm{H}), 1.38-1.09(\mathrm{~m}, 18 \mathrm{H}), 0.94-0.68(\mathrm{~m}, 4 \mathrm{H}) .{ }^{13} \mathrm{C}\left\{{ }^{1} \mathrm{H}\right\} \mathrm{NMR}\left(63 \mathrm{MHz}, \delta, \mathrm{CDCl}_{3}\right): 82.8$, $39.9,32.9,31.4,26.8,26.5,24.8$.

2-(3,3-dimethylbutyl)-4,4,5,5-tetramethyl-1,3,2-dioxaborolane: ${ }^{[3]}$ colorless oil, ${ }^{1} \mathrm{H} N M R(250 \mathrm{MHz}, \delta$, $\left.\mathrm{CDCl}_{3}\right): 1.43-1.22(\mathrm{~m}, 14 \mathrm{H}), 1.02-0.83(\mathrm{~m}, 9 \mathrm{H}), 0.72(\mathrm{t}, \mathrm{J}=9.7 \mathrm{~Hz}, 2 \mathrm{H}) .{ }^{13} \mathrm{C}\left\{{ }^{1} \mathrm{H}\right\} \mathrm{NMR}\left(63 \mathrm{MHz}, \delta, \mathrm{CDCl}_{3}\right)$ : $82.8,37.7,30.8,28.8,24.8$.

2-(2-chlorophenethyl)-4,4,5,5-tetramethyl-1,3,2-dioxaborolane: ${ }^{[4]}$ colorless oil, ${ }^{1} \mathrm{H} \mathrm{NMR}(400 \mathrm{MHz}, \delta$, $\left.\mathrm{CDCl}_{3}\right): 7.32(\mathrm{~d}, J=7.7 \mathrm{~Hz}, 1 \mathrm{H}), 7.28(\mathrm{~d}, J=7.5 \mathrm{~Hz}, 1 \mathrm{H}), 7.19(\mathrm{td}, J=7.5,1.5 \mathrm{~Hz}, 1 \mathrm{H}), 7.12(\mathrm{td}, J=7.6,1.9$ $\mathrm{Hz}, 1 \mathrm{H}), 2.87(\mathrm{t}, J=8.2 \mathrm{~Hz}, 2 \mathrm{H}), 1.26(\mathrm{~s}, 12 \mathrm{H}), 1.17(\mathrm{t}, J=8.2 \mathrm{~Hz}, 2 \mathrm{H}) .{ }^{13} \mathrm{C}\left\{{ }^{1} \mathrm{H}\right\} \mathrm{NMR}\left(101 \mathrm{MHz}, \delta, \mathrm{CDCl}_{3}\right)$ : $141.8,133.8,129.5$ (d, J = $45 \mathrm{~Hz}), 126.8(\mathrm{~d}, J=36 \mathrm{~Hz}), 83.1,27.8,24.8$. 
4,4,5,5-tetramethyl-2-(3-phenoxypropyl)-1,3,2-dioxaborolane: ${ }^{[5]}$ colorless oil, ${ }^{1} \mathrm{H}$ NMR (400 MHz, $\delta$, $\mathrm{CD}_{2} \mathrm{Cl}_{2}$ ): $7.30(\mathrm{dd}, J=8.7,7.3 \mathrm{~Hz}, 2 \mathrm{H}), 6.98-6.90(\mathrm{~m}, 3 \mathrm{H}), 3.97(\mathrm{t}, J=6.7 \mathrm{~Hz}, 2 \mathrm{H}$ ), 1.89 (qui, $J=7.5 \mathrm{~Hz}$, $2 \mathrm{H}), 1.27(\mathrm{~s}, 12 \mathrm{H}), 0.92(\mathrm{t}, J=7.8 \mathrm{~Hz}, 2 \mathrm{H}) .{ }^{13} \mathrm{C}\left\{{ }^{1} \mathrm{H}\right\} \mathrm{NMR}\left(101 \mathrm{MHz}, \delta, \mathrm{CD}_{2} \mathrm{Cl}_{2}\right): 159.2,129.3,120.2$, $114.4,83.0,69.5,24.6,23.7$.

2-(2-(cyclohex-3-en-1-yl)ethyl)-4,4,5,5-tetramethyl-1,3,2-dioxaborolane: ${ }^{[2]}$ colorless oil, ${ }^{1} \mathrm{H}$ NMR (400 $\left.\mathrm{MHz}, \delta, \mathrm{CDCl}_{3}\right): 5.66(\mathrm{~m}, 2 \mathrm{H}), 2.16-1.99(\mathrm{~m}, 3 \mathrm{H}), 1.89-1.72(\mathrm{~m}, 1 \mathrm{H}), 1.69-1.58(\mathrm{~m}, 1 \mathrm{H}), 1.51-1.35(\mathrm{~m}$, $4 \mathrm{H}), 1.26(\mathrm{~s}, 12 \mathrm{H}), 0.81(\mathrm{t}, J=6.8 \mathrm{~Hz}, 2 \mathrm{H}) .{ }^{13} \mathrm{C}\left\{{ }^{1} \mathrm{H}\right\} \operatorname{NMR}\left(101 \mathrm{MHz}, \delta, \mathrm{CDCl}_{3}\right): 127.0,126.7,82.8,35.8$, $31.6,30.6,28.5 .25 .3,24.8$.

4,4,5,5-tetramethyl-2-(2-(perfluorophenyl)ethyl)-1,3,2-dioxaborolane: ${ }^{[5]}$ colorless oil, ${ }^{1} \mathrm{H}$ NMR (250 $\left.\mathrm{MHz}, \delta, \mathrm{CD}_{2} \mathrm{Cl}_{2}\right): 2.82(\mathrm{t}, J=7.7 \mathrm{~Hz}, 2 \mathrm{H}), 1.25(\mathrm{~s}, 12 \mathrm{H}), 1.13(\mathrm{t}, J=7.7 \mathrm{~Hz}, 2 \mathrm{H}) .{ }^{13} \mathrm{C}\left\{{ }^{1} \mathrm{H}\right\} \mathrm{NMR}(63 \mathrm{MHz}, \delta$, $\left.\mathrm{CD}_{2} \mathrm{Cl}_{2}\right): 128.2,83.3,24.5,16.9$.

2-dodecyl-4,4,5,5-tetramethyl-1,3,2-dioxaborolane: ${ }^{[6]}$ colorless oil, ${ }^{1} \mathrm{H} \mathrm{NMR}\left(250 \mathrm{MHz}, \delta, \mathrm{CD}_{2} \mathrm{Cl}_{2}\right)$ : 1.40-1.18 (m, 32H), $0.88(\mathrm{t}, J=7.1 \mathrm{~Hz}, 3 \mathrm{H}), 0.76(\mathrm{t}, J=7.8 \mathrm{~Hz}, 2 \mathrm{H}) .{ }^{13} \mathrm{C}\left\{{ }^{1} \mathrm{H}\right\} \mathrm{NMR}\left(63 \mathrm{MHz}, \delta, \mathrm{CD}_{2} \mathrm{Cl}_{2}\right)$ : $82.6,32.4,31.9,29.6,29.4(\mathrm{~d}, J=6.0 \mathrm{~Hz}), 24.6,24.0,22.7,13.8$. 


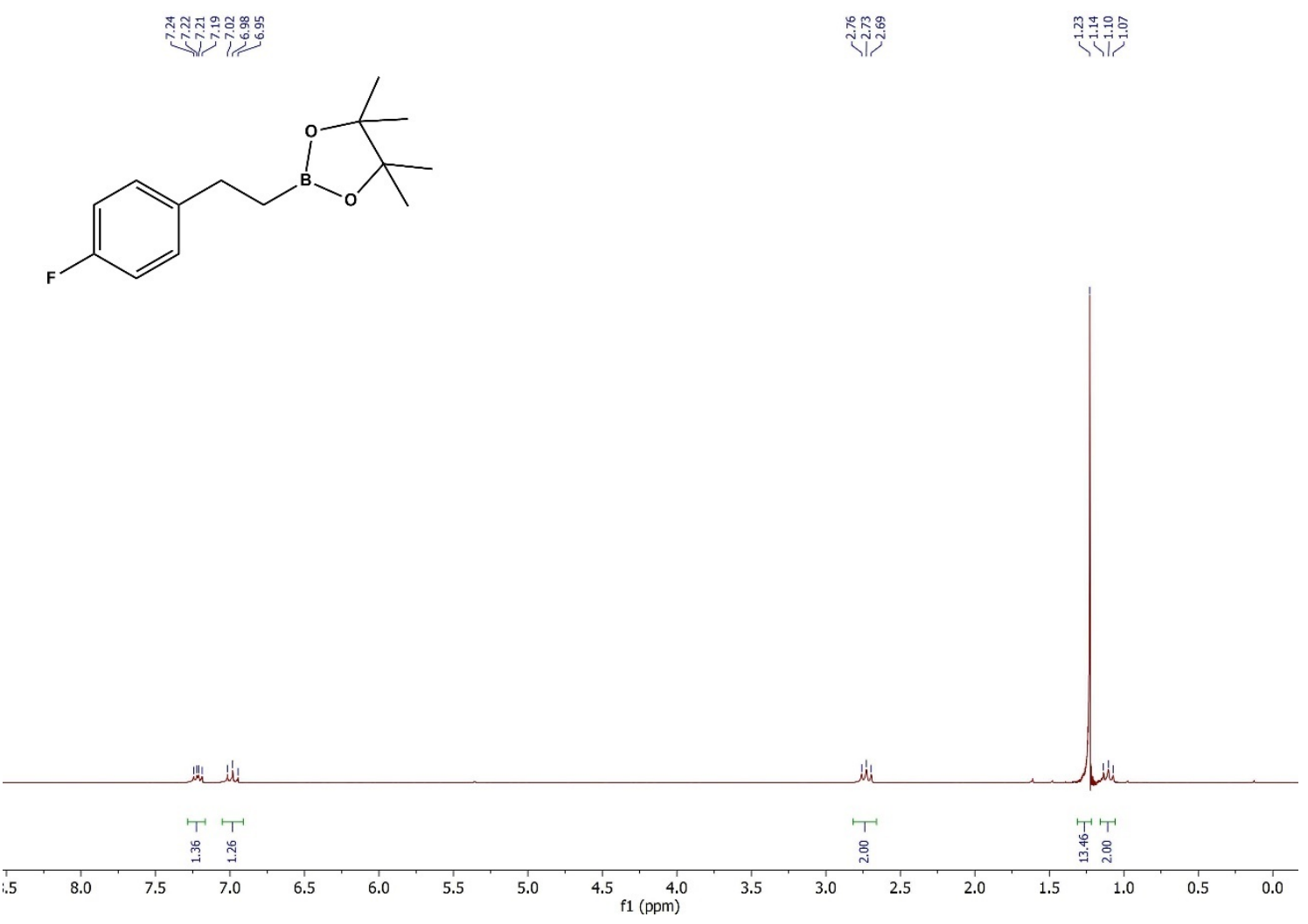

Figure S15. ${ }^{1} \mathrm{H}$ NMR Spectrum of 2-(4-fluorophenethyl)-4,4,5,5-tetramethyl-1,3,2-dioxaborolane.
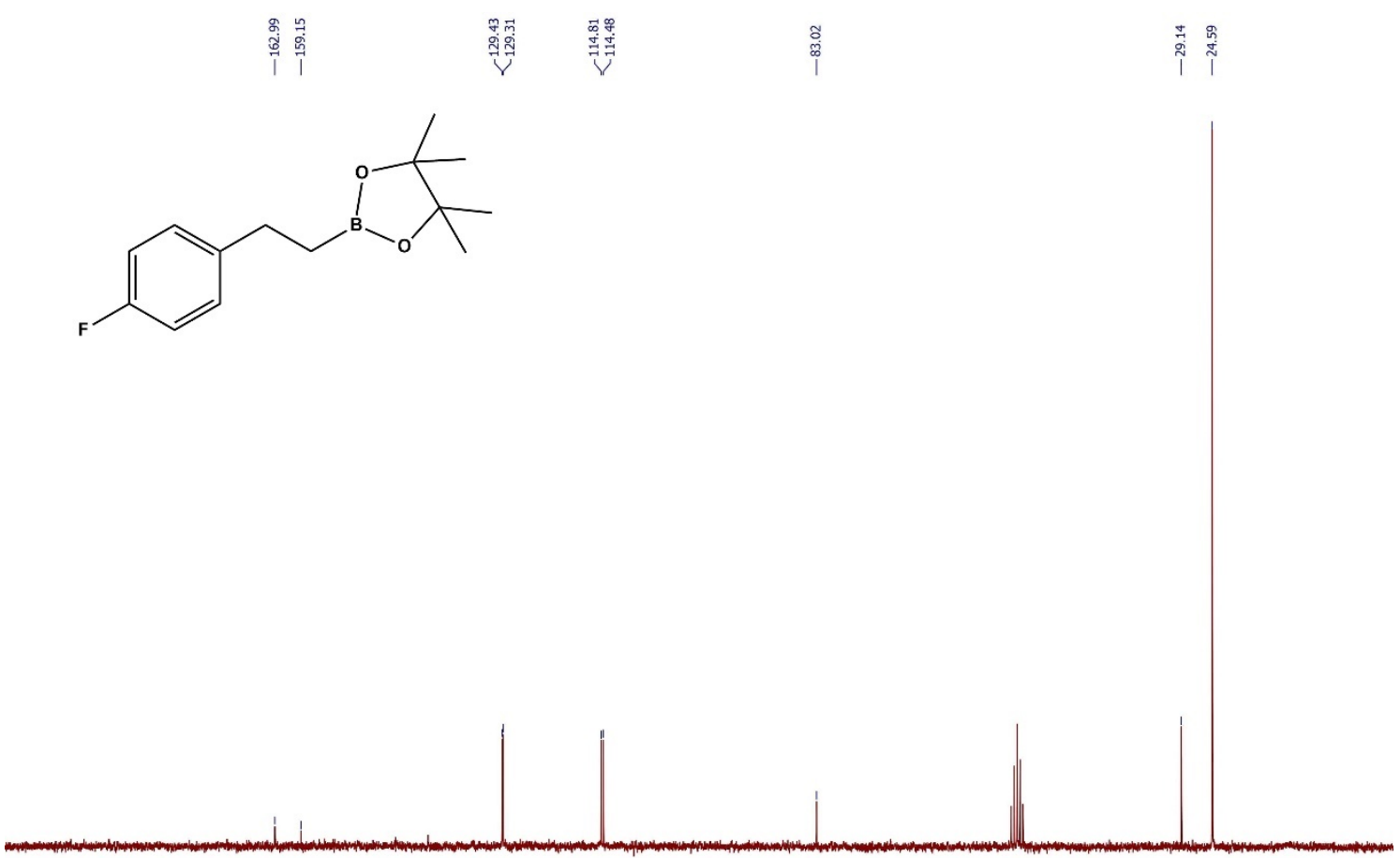

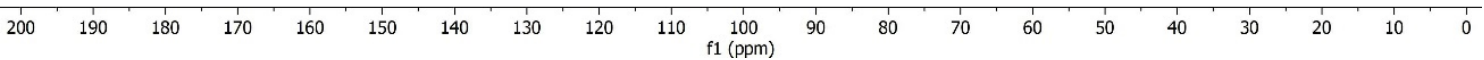

Figure S16. ${ }^{13} \mathrm{C}$ NMR Spectrum of 2-(4-fluorophenethyl)-4,4,5,5-tetramethyl-1,3,2-dioxaborolane. 


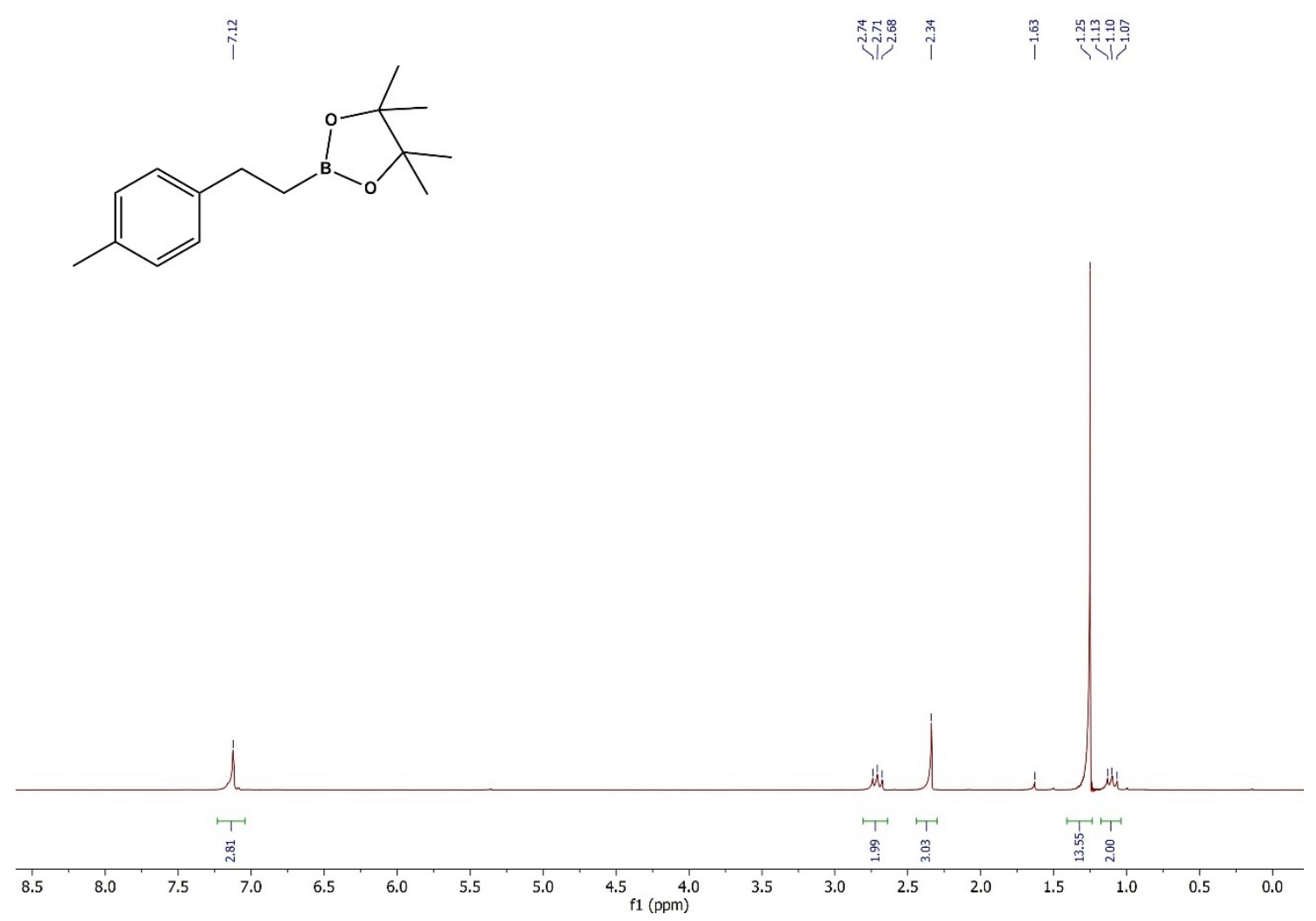

Figure S17. ${ }^{1} \mathrm{H}$ NMR Spectrum of 4,4,5,5-tetramethyl-2-(4-methylphenethyl)-1,3,2-dioxaborolane.

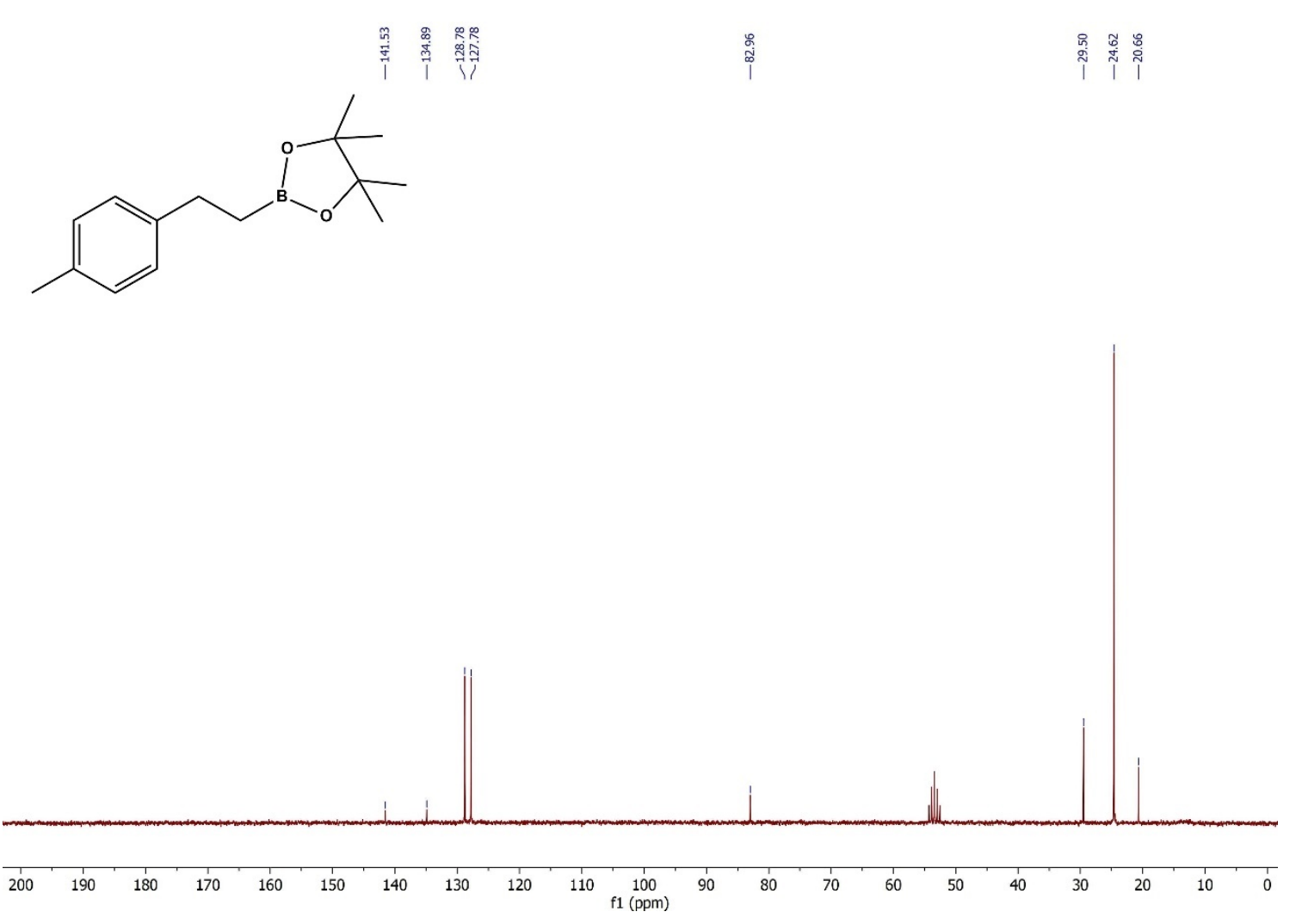

Figure S18. ${ }^{13} \mathrm{C}$ NMR Spectrum of 4,4,5,5-tetramethyl-2-(4-methylphenethyl)-1,3,2-dioxaborolane. 


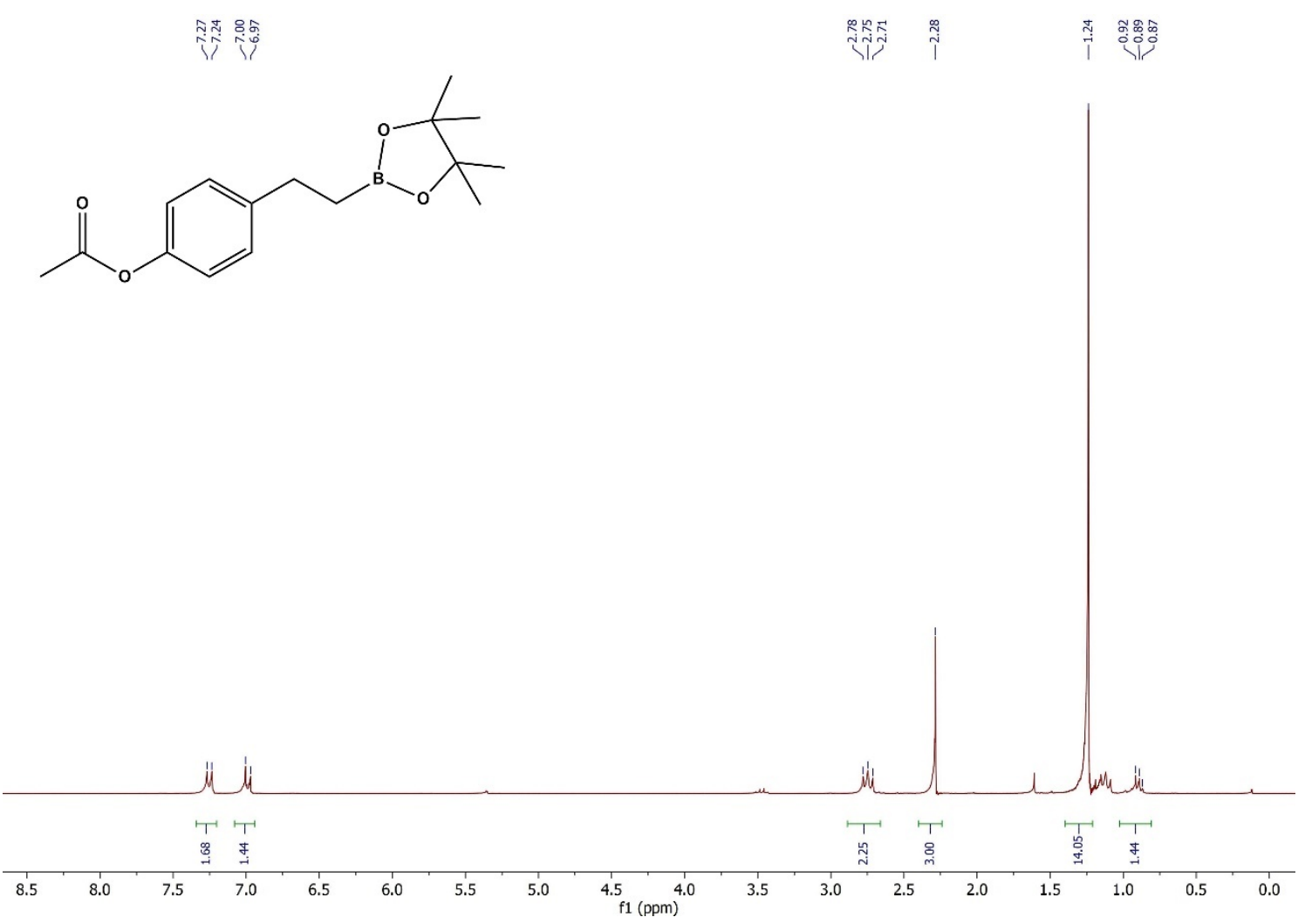

Figure S19. ${ }^{1} \mathrm{H}$ NMR Spectrum of 4-(2-(4,4,5,5-tetramethyl-1,2,3-dioxaborolan-2-yl)ethyl)phenyl acetate.
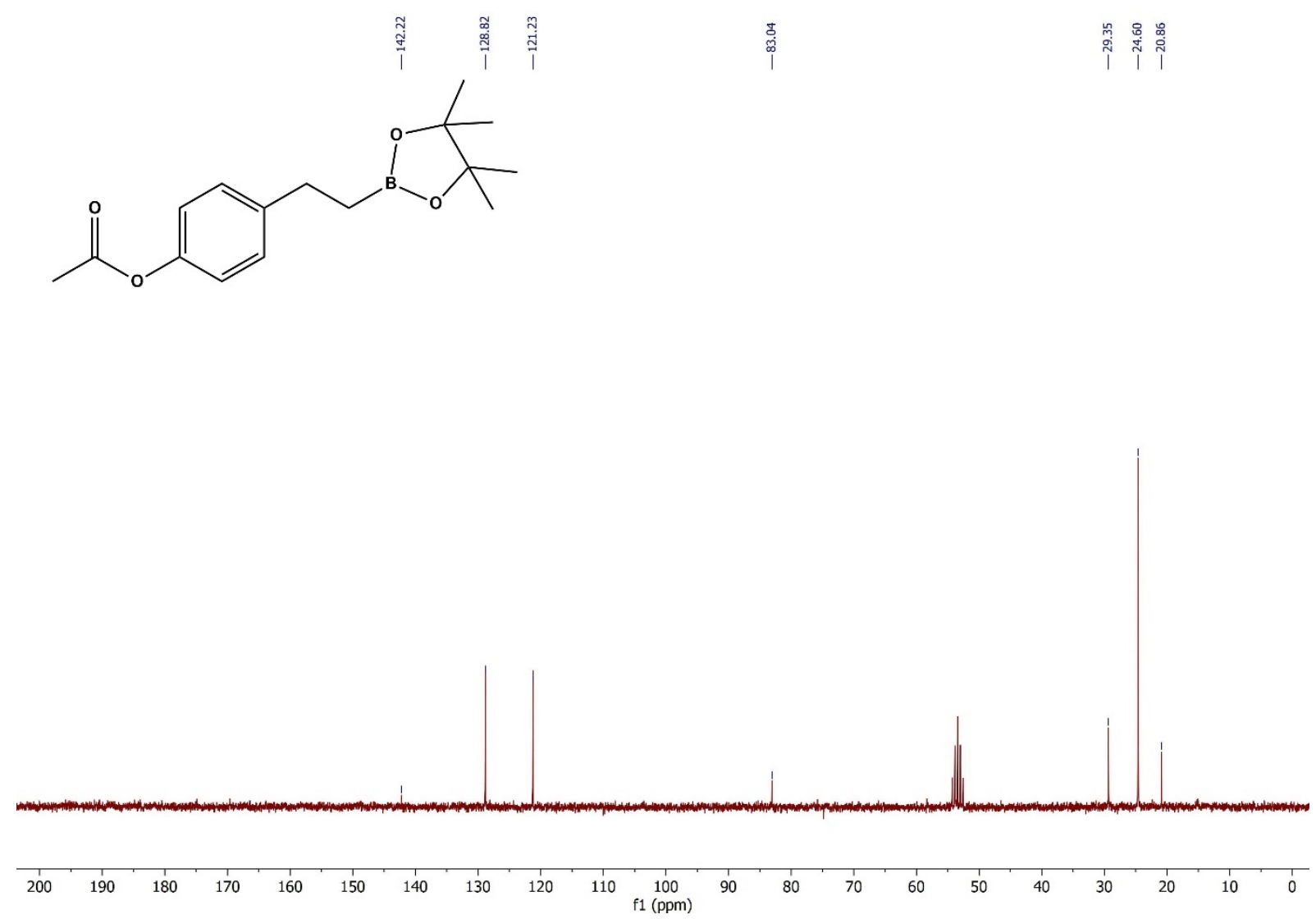

Figure S20. ${ }^{13} \mathrm{C}$ NMR Spectrum of 4-(2-(4,4,5,5-tetramethyl-1,2,3-dioxaborolan-2-yl)ethyl)phenyl acetate. 


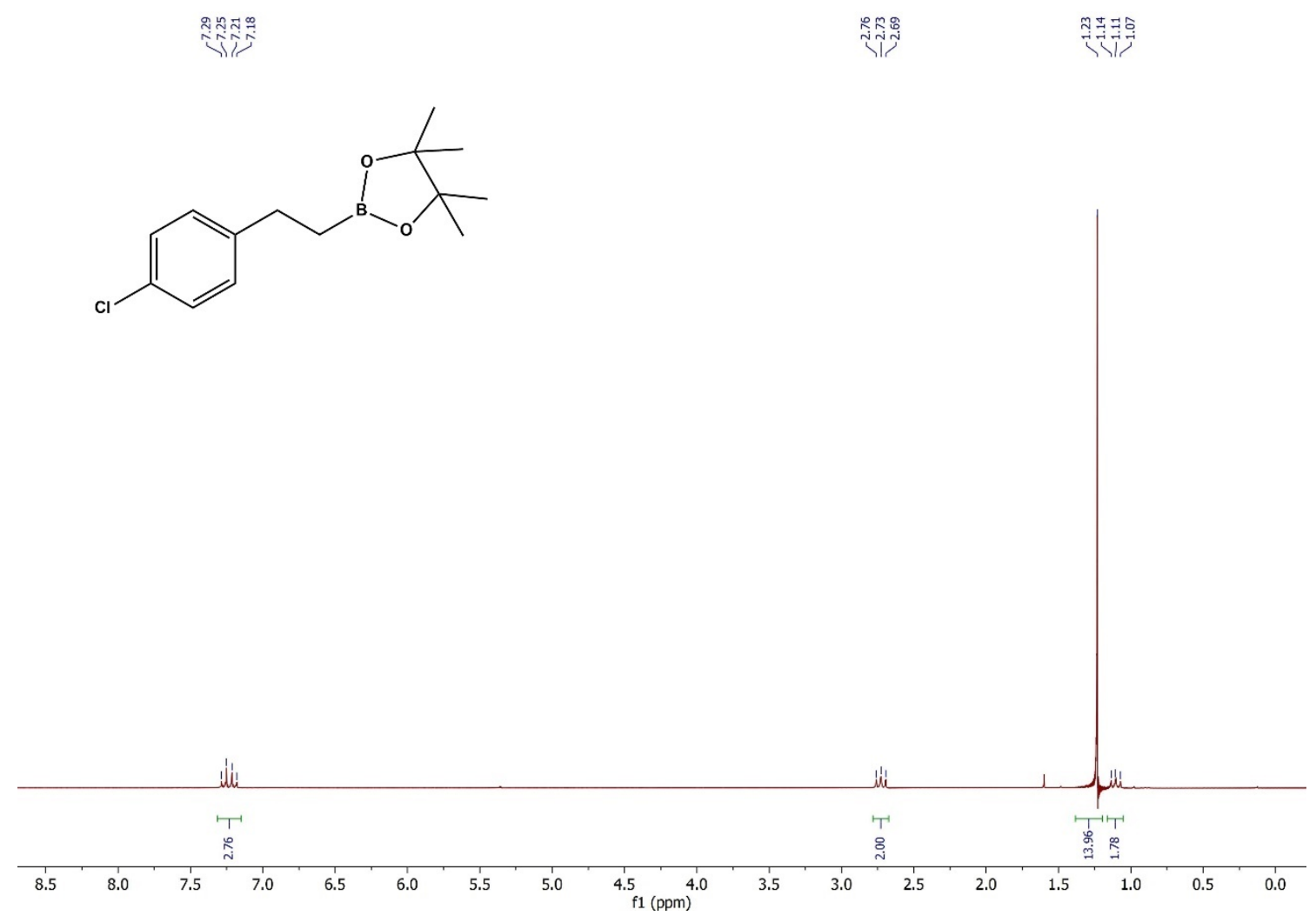

Figure S21. ${ }^{1} \mathrm{H}$ NMR Spectrum of 2-(4-chlorophenethyl)-4,4,5,5-tetramethyl-1,3,2-dioxaborolane.

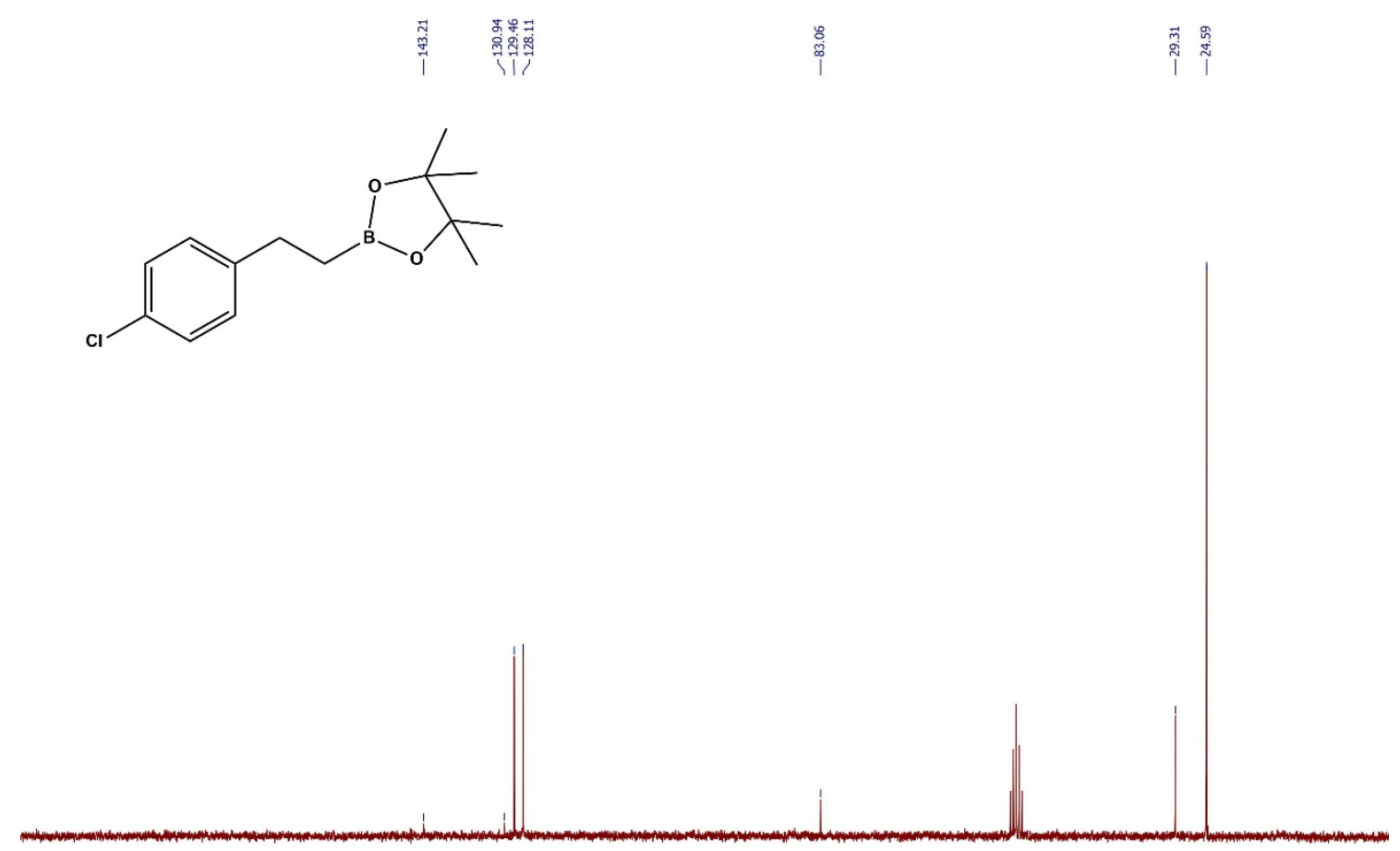

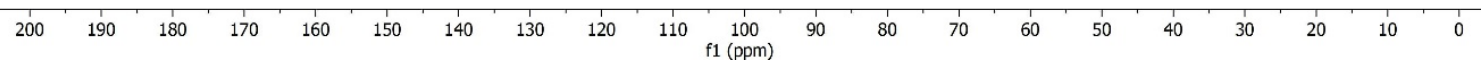

Figure S22. ${ }^{13} \mathrm{C}$ NMR Spectrum of 2-(4-chlorophenethyl)-4,4,5,5-tetramethyl-1,3,2-dioxaborolane. 

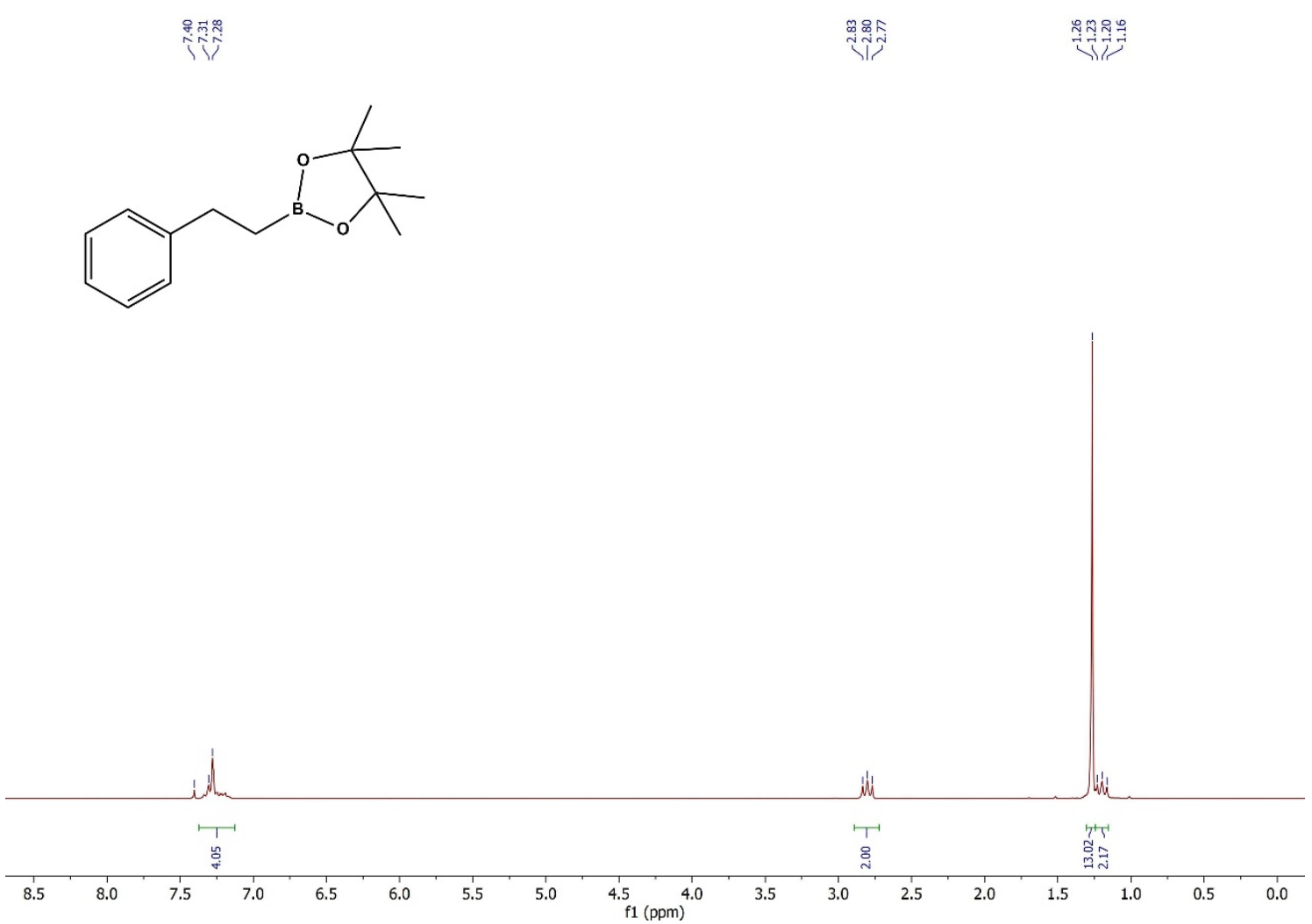

Figure S23. ${ }^{1} \mathrm{H}$ NMR Spectrum of 4,4,5,5,-tetramethyl-2-phenethyl-1,3,2-dioxaborolane.
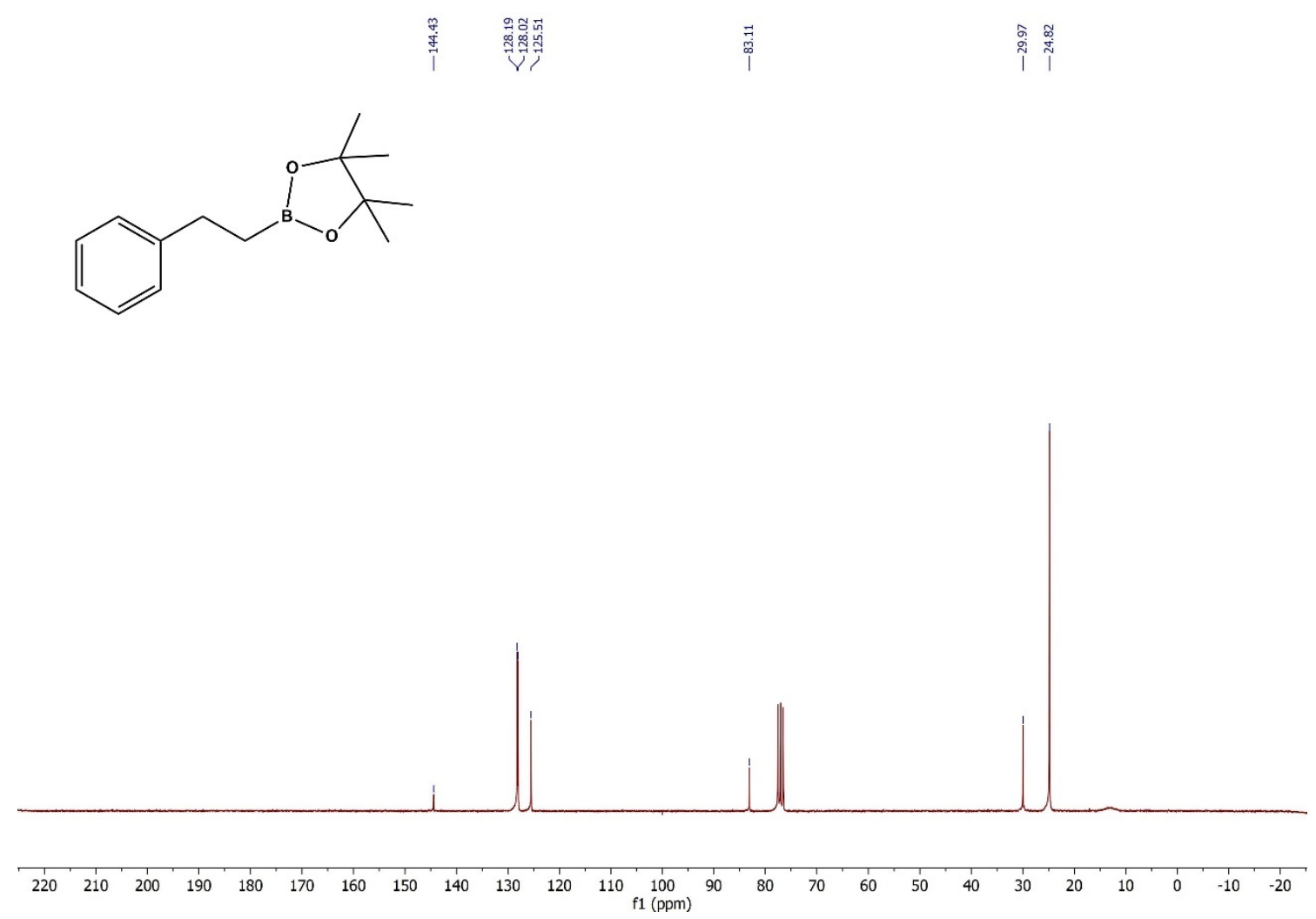

Figure S24. ${ }^{13} \mathrm{C}$ NMR Spectrum of 4,4,5,5,-tetramethyl-2-phenethyl-1,3,2-dioxaborolane. 

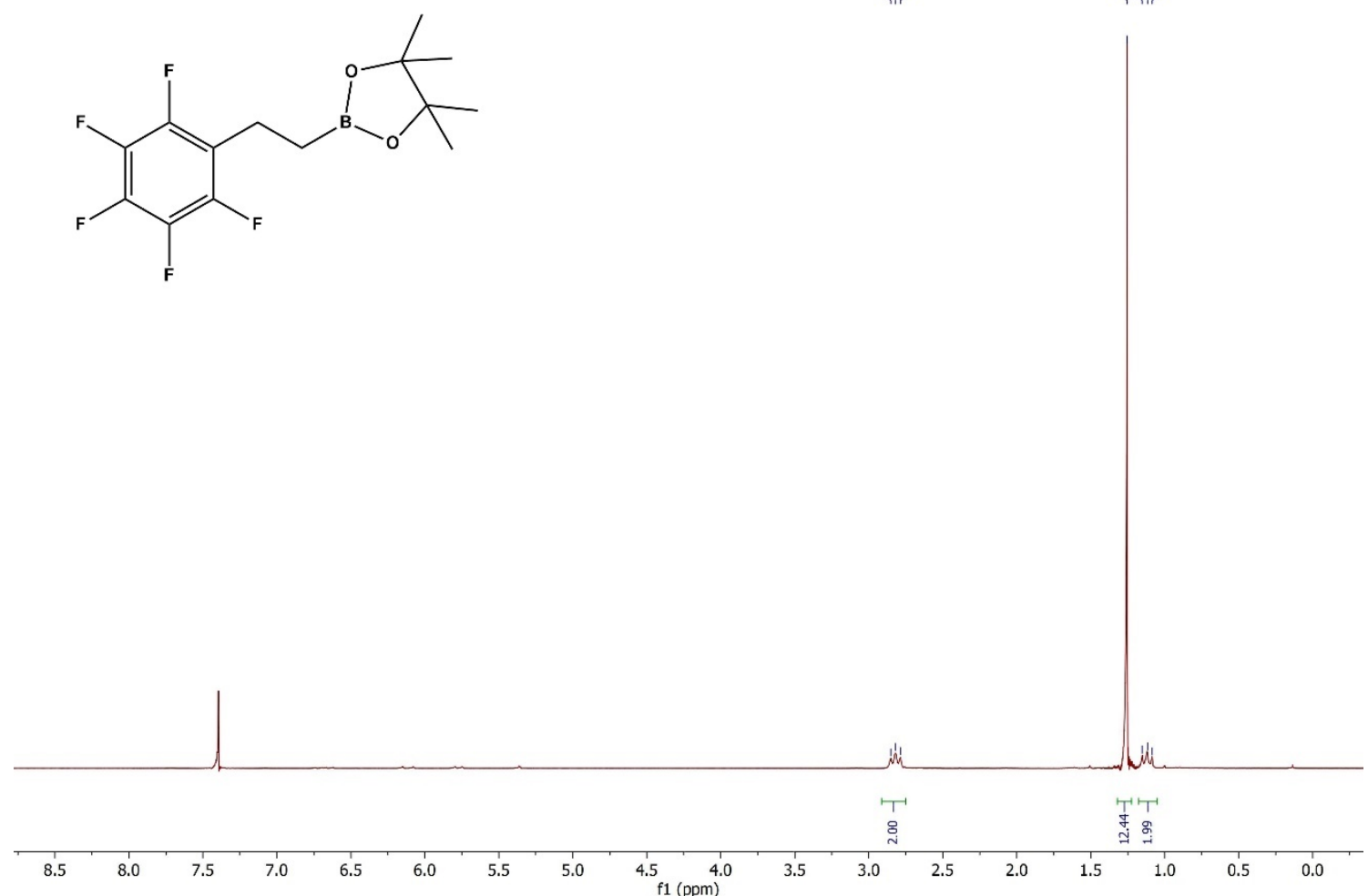

Figure S25. ${ }^{1} \mathrm{H}$ NMR Spectrum of 4,4,5,5-tetramethyl-2-(2-(perfluorophenyl)ethyl)-1,3,2dioxaborolane.<smiles>CC1(C)OB(CCc2c(F)c(F)c(F)c(F)c2F)OC1(C)C</smiles>

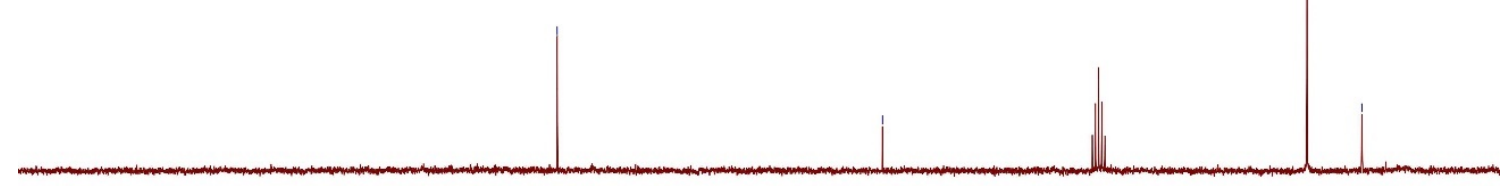

Figure S26. ${ }^{13} \mathrm{C}$ NMR Spectrum of 4,4,5,5-tetramethyl-2-(2-(perfluorophenyl)ethyl)-1,3,2dioxaborolane. 


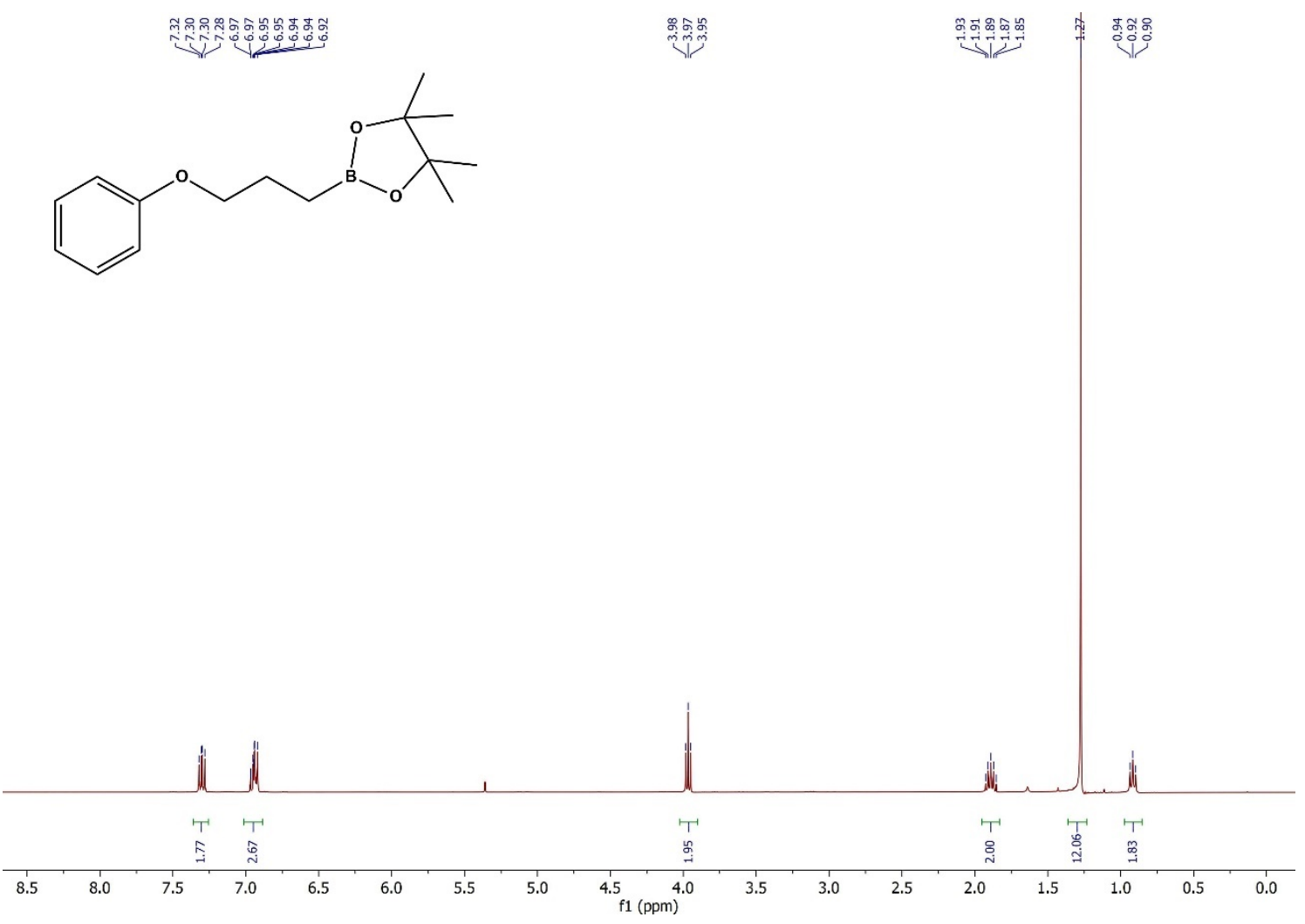

Figure S27. ${ }^{1} \mathrm{H}$ NMR Spectrum of 4,4,5,5-tetramethyl-2-(3-phenoxypropyl)-1,3,2-dioxaborolane.
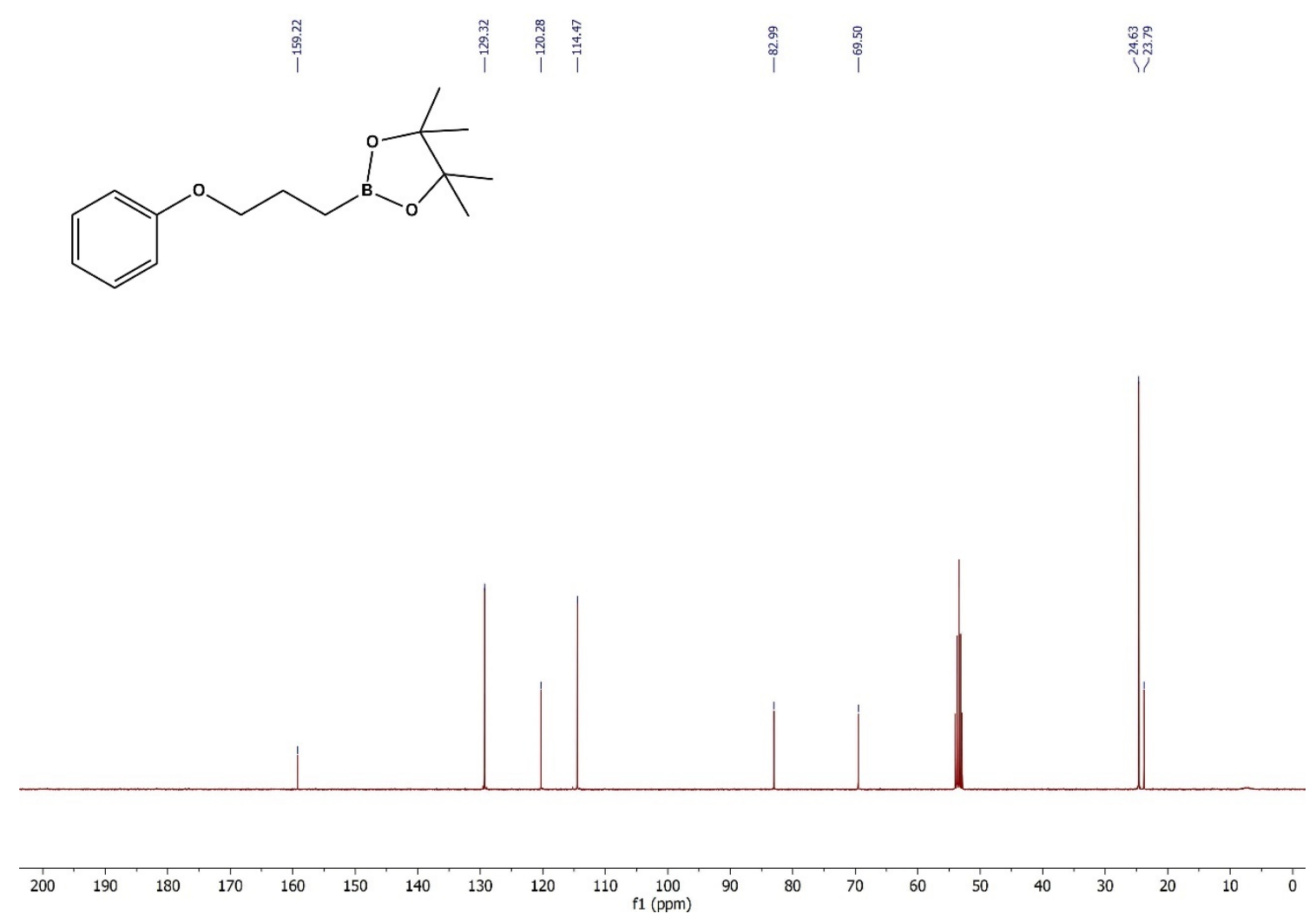

Figure S28. ${ }^{13} \mathrm{C}$ NMR Spectrum of 4,4,5,5-tetramethyl-2-(3-phenoxypropyl)-1,3,2-dioxaborolane. 


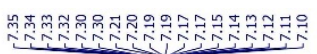<smiles>CC1(C)OB(CCc2ccccc2Cl)OC1(C)C</smiles>
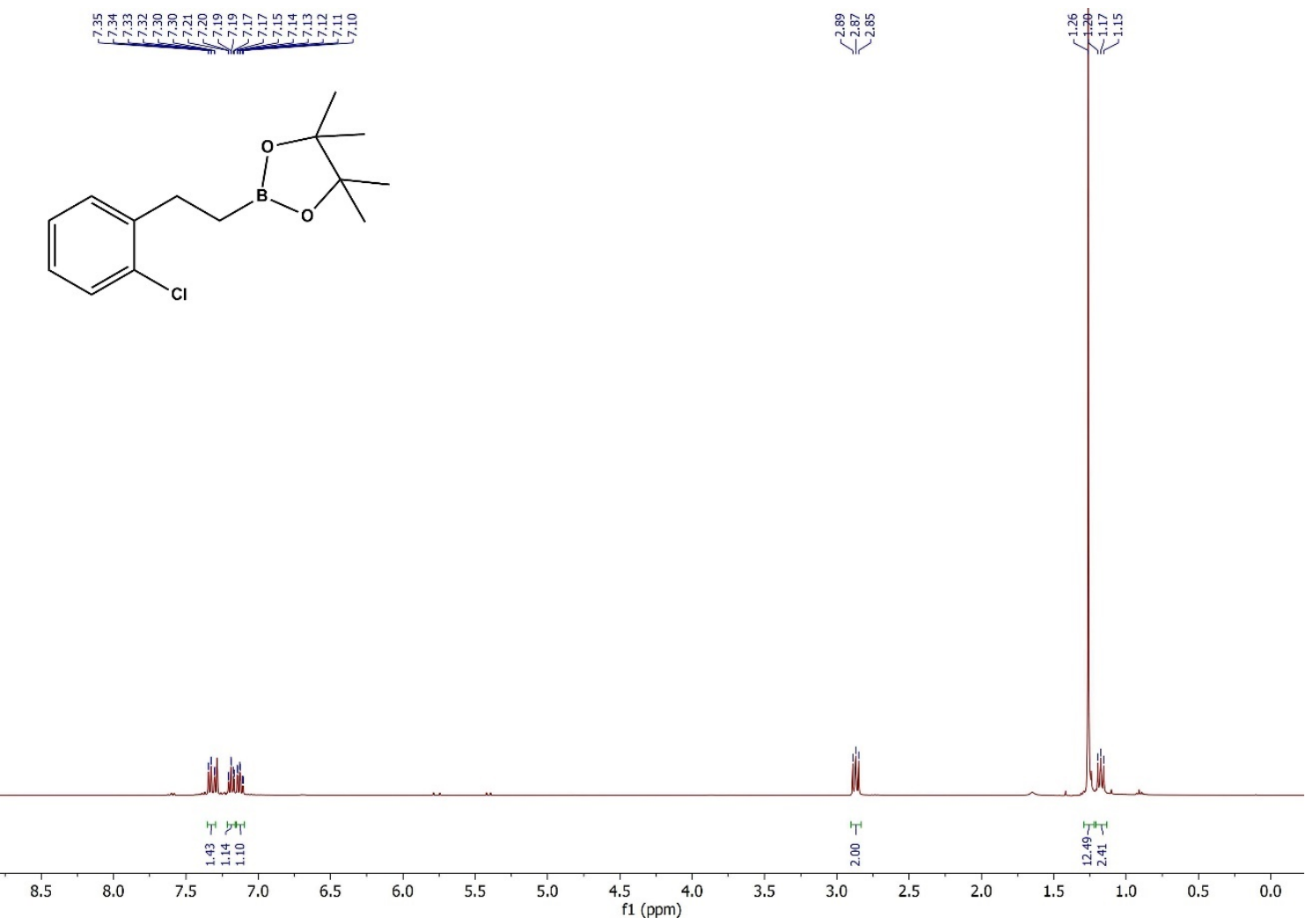

Figure S29. ${ }^{1} \mathrm{H}$ NMR Spectrum of 2-(2-chlorophenethyl)-4,4,5,5-tetramethyl-1,3,2-dioxaborolane.
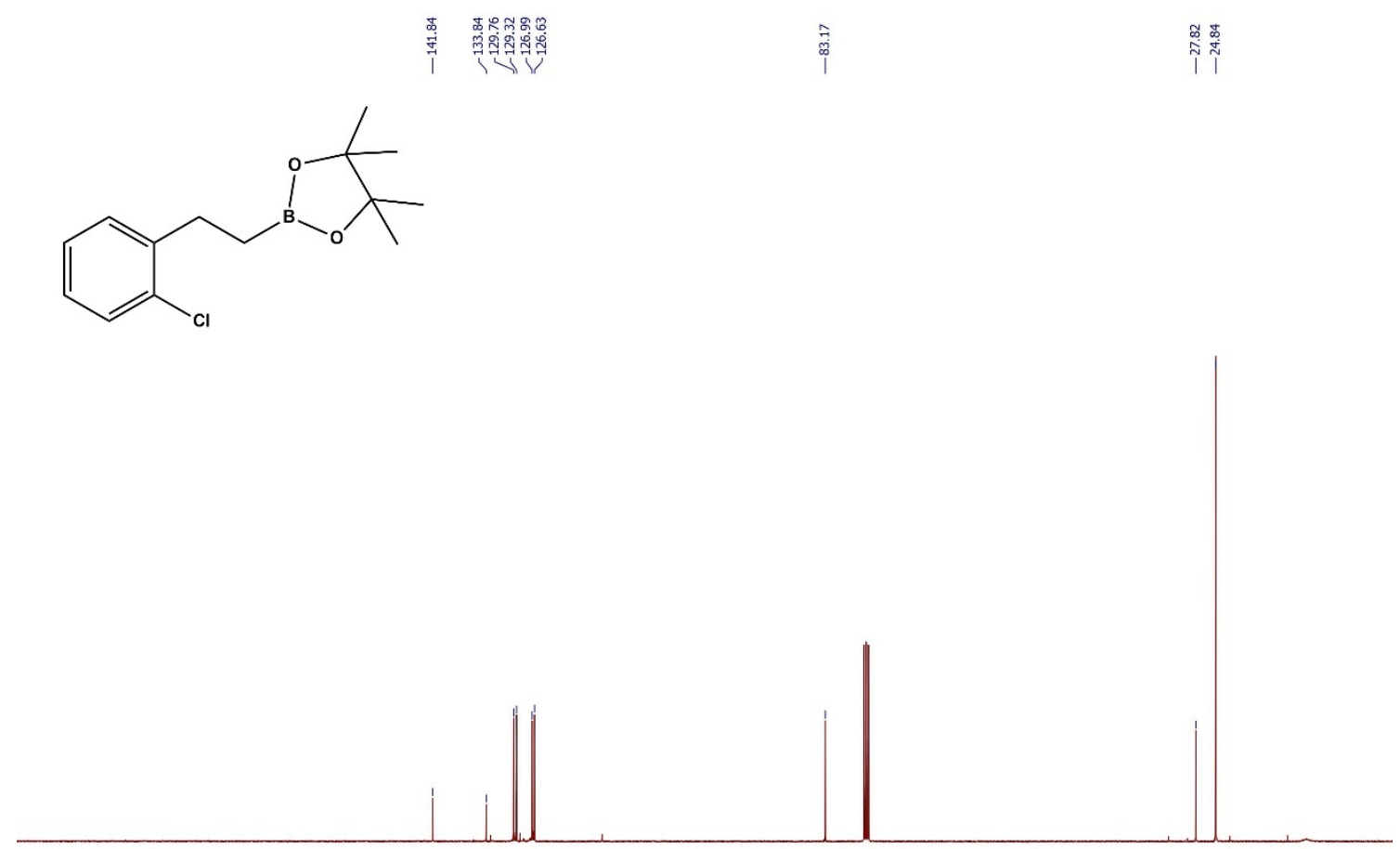

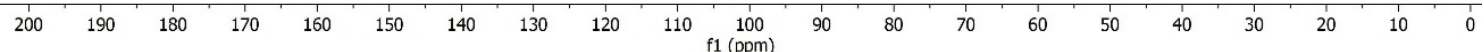

Figure S30. ${ }^{13} \mathrm{C}$ NMR Spectrum of 2-(2-chlorophenethyl)-4,4,5,5-tetramethyl-1,3,2-dioxaborolane. 


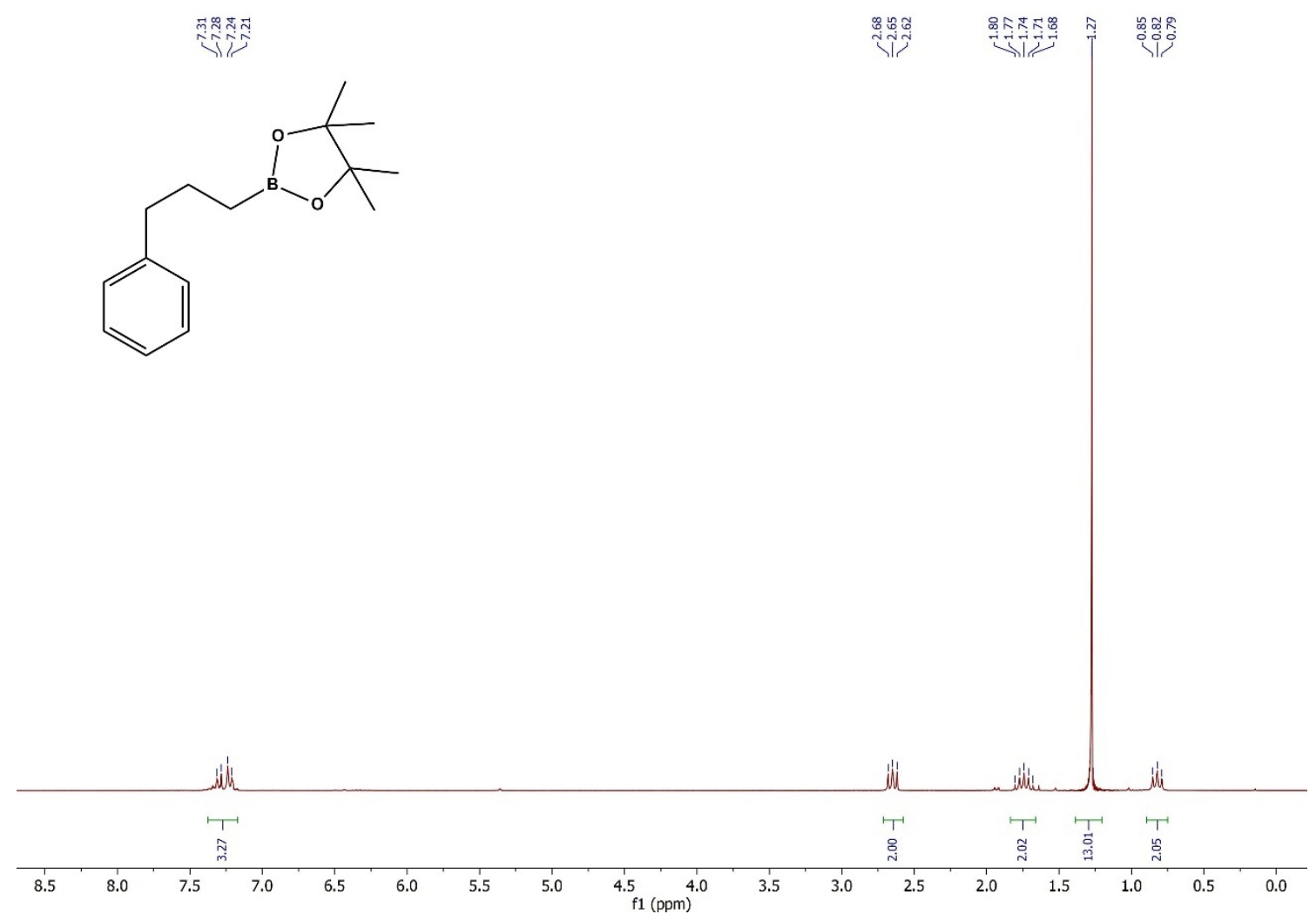

Figure S31. ${ }^{1} \mathrm{H}$ NMR Spectrum of 4,4,5,5-tetramethyl-2-(3-phenylpropyl)-1,3,2-dioxaborolane.

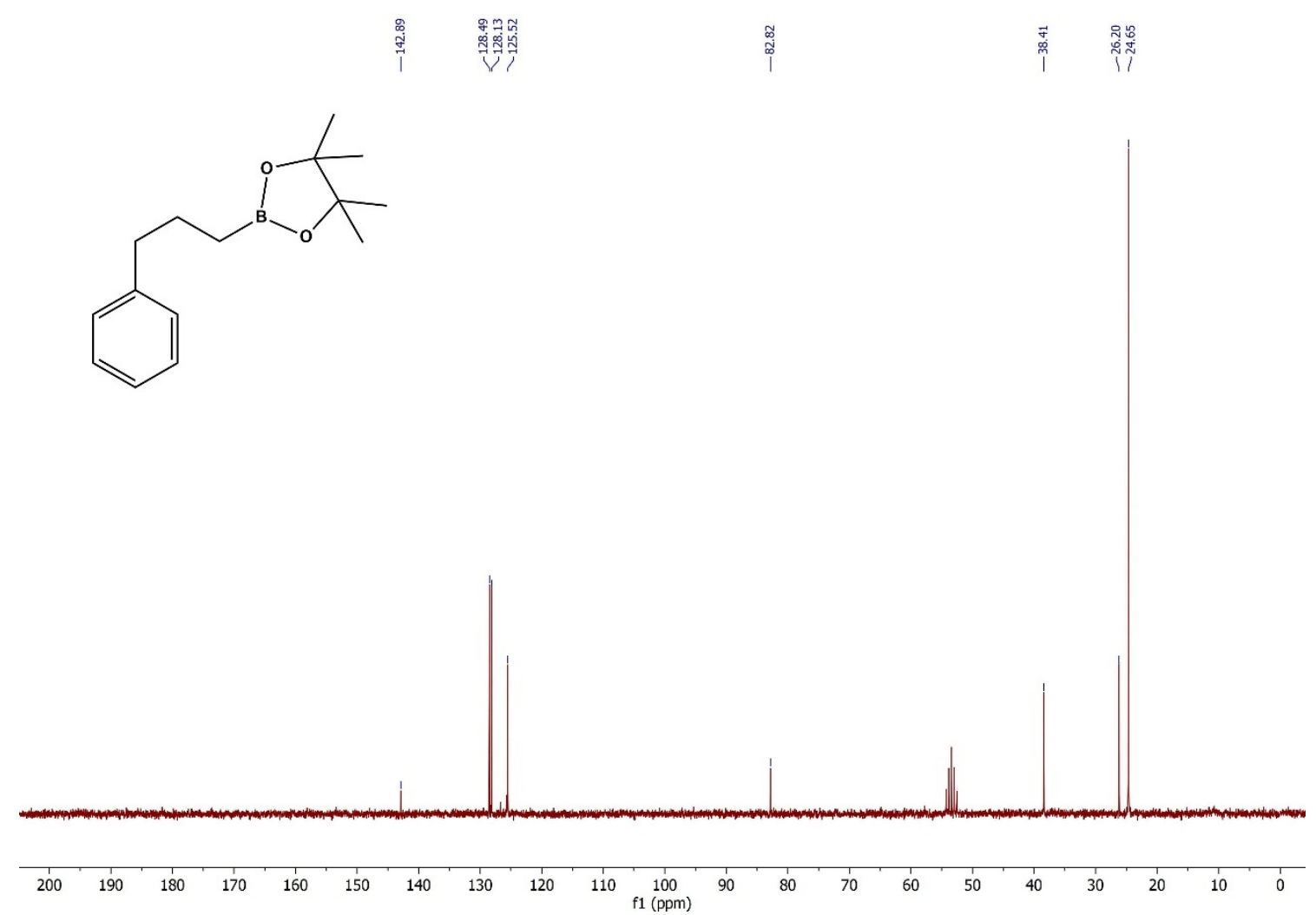

Figure S32. ${ }^{13} \mathrm{C}$ NMR Spectrum of 4,4,5,5-tetramethyl-2-(3-phenylpropyl)-1,3,2-dioxaborolane. 


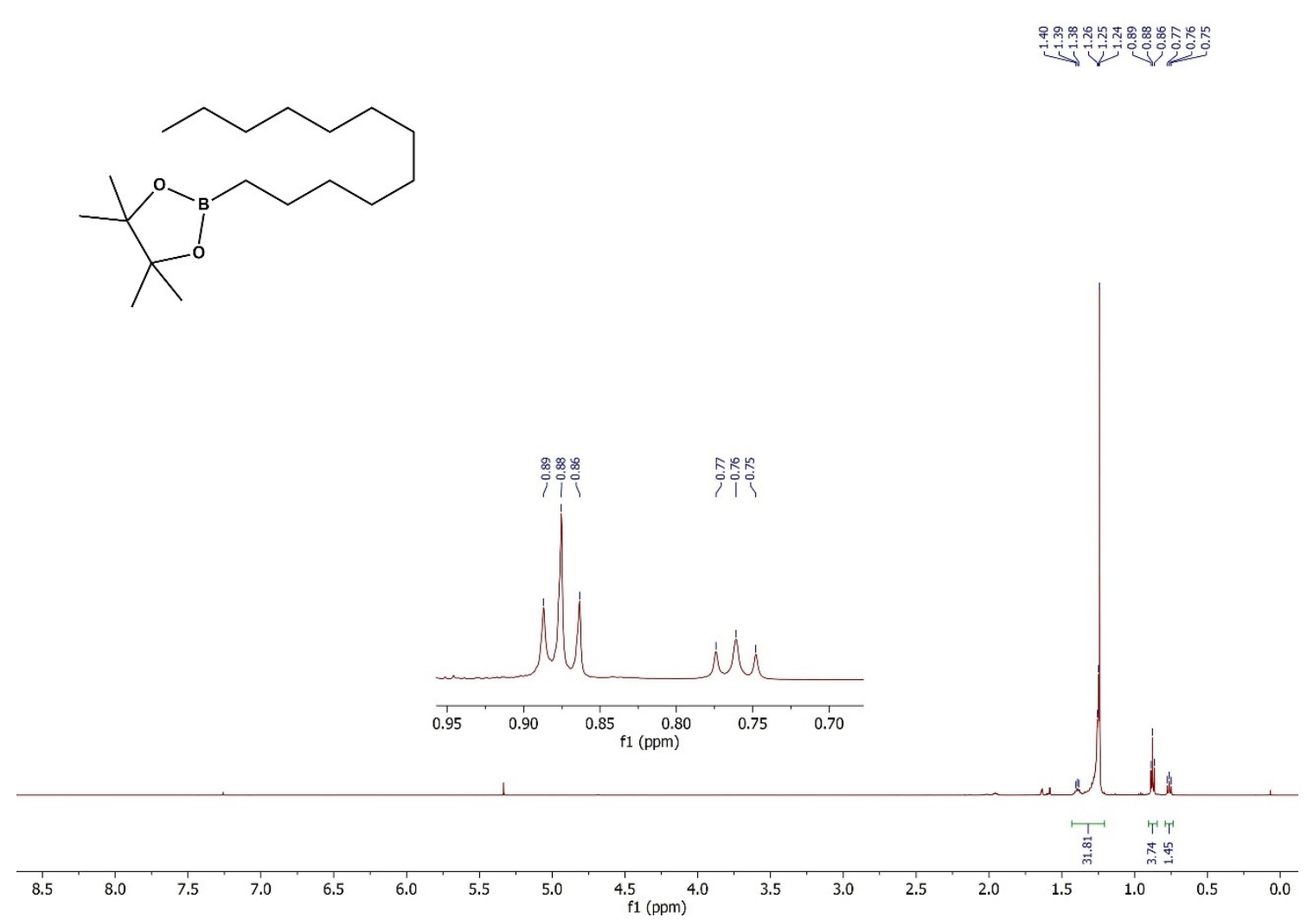

Figure S33. ${ }^{1} \mathrm{H}$ NMR Spectrum of 2-dodecyl-4,4,5,5-tetramethyl-1,3,2-dioxaborolane.
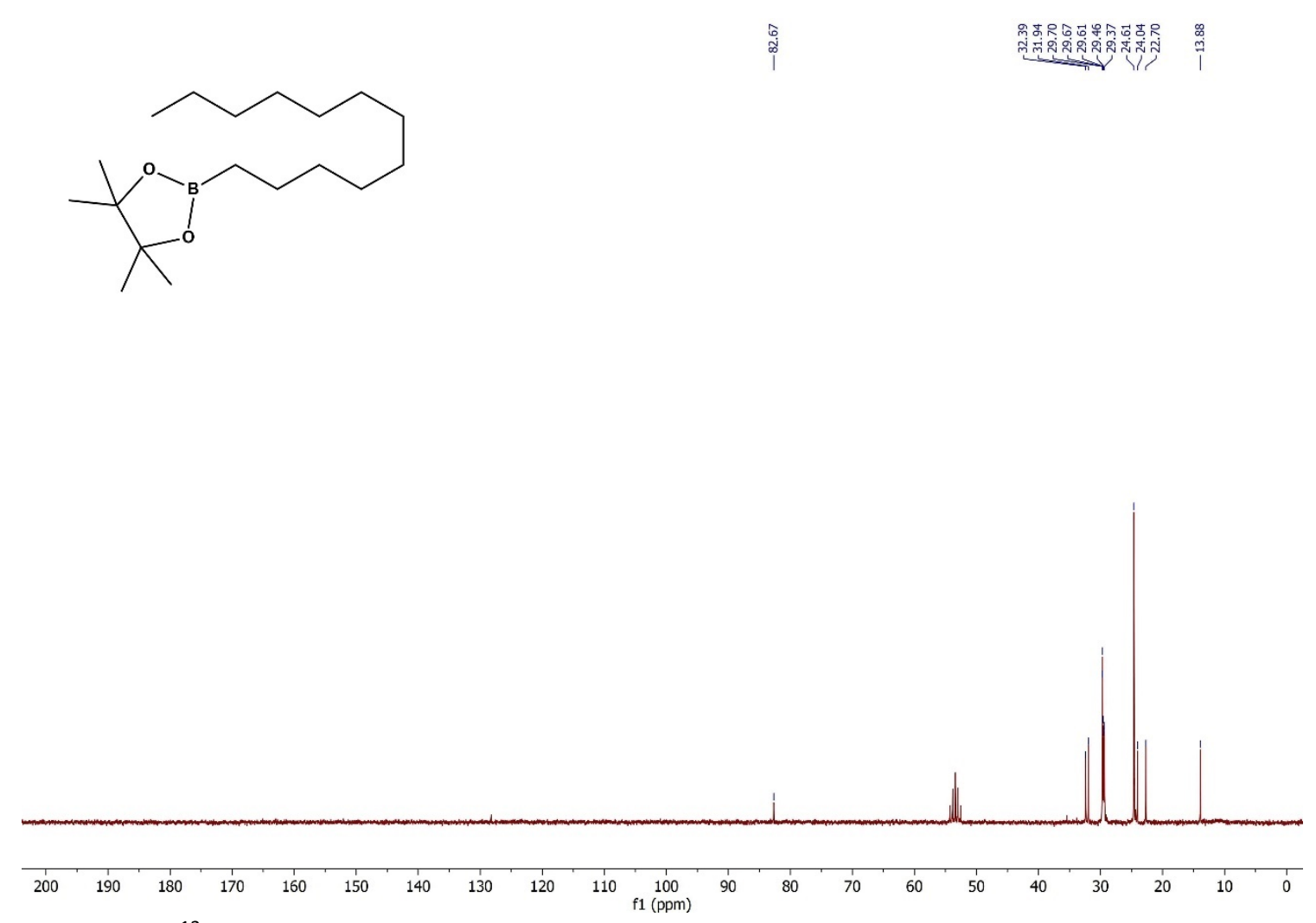

Figure S34. ${ }^{13} \mathrm{C}$ NMR Spectrum of 2-dodecyl-4,4,5,5-tetramethyl-1,3,2-dioxaborolane. 


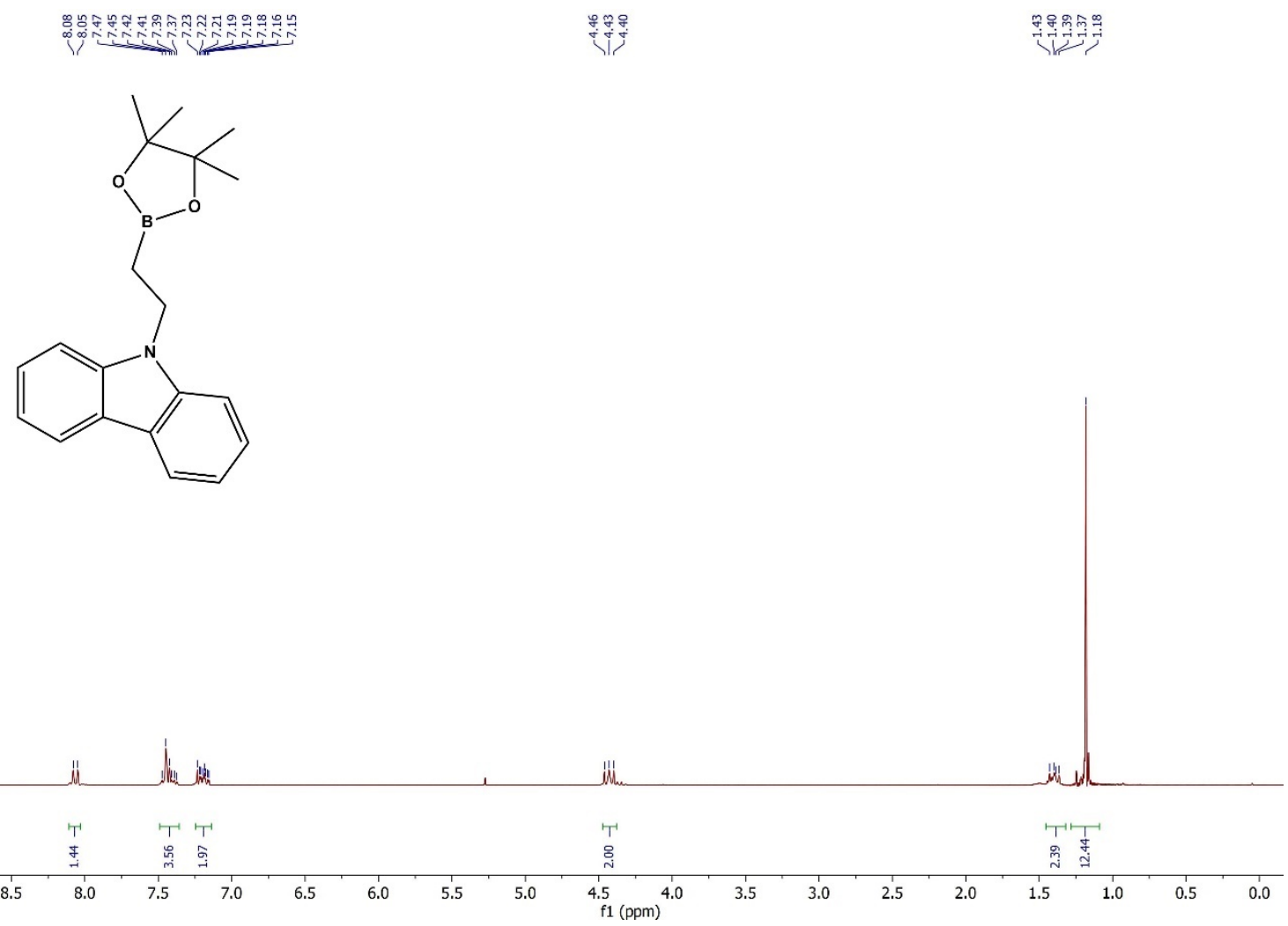

Figure S35. ${ }^{1} \mathrm{H}$ NMR Spectrum of 9-(2-(4,4,5,5-tetramethyl-1,3,2-dioxaborolan-2-yl)ethyl)-9Hcarbazole.
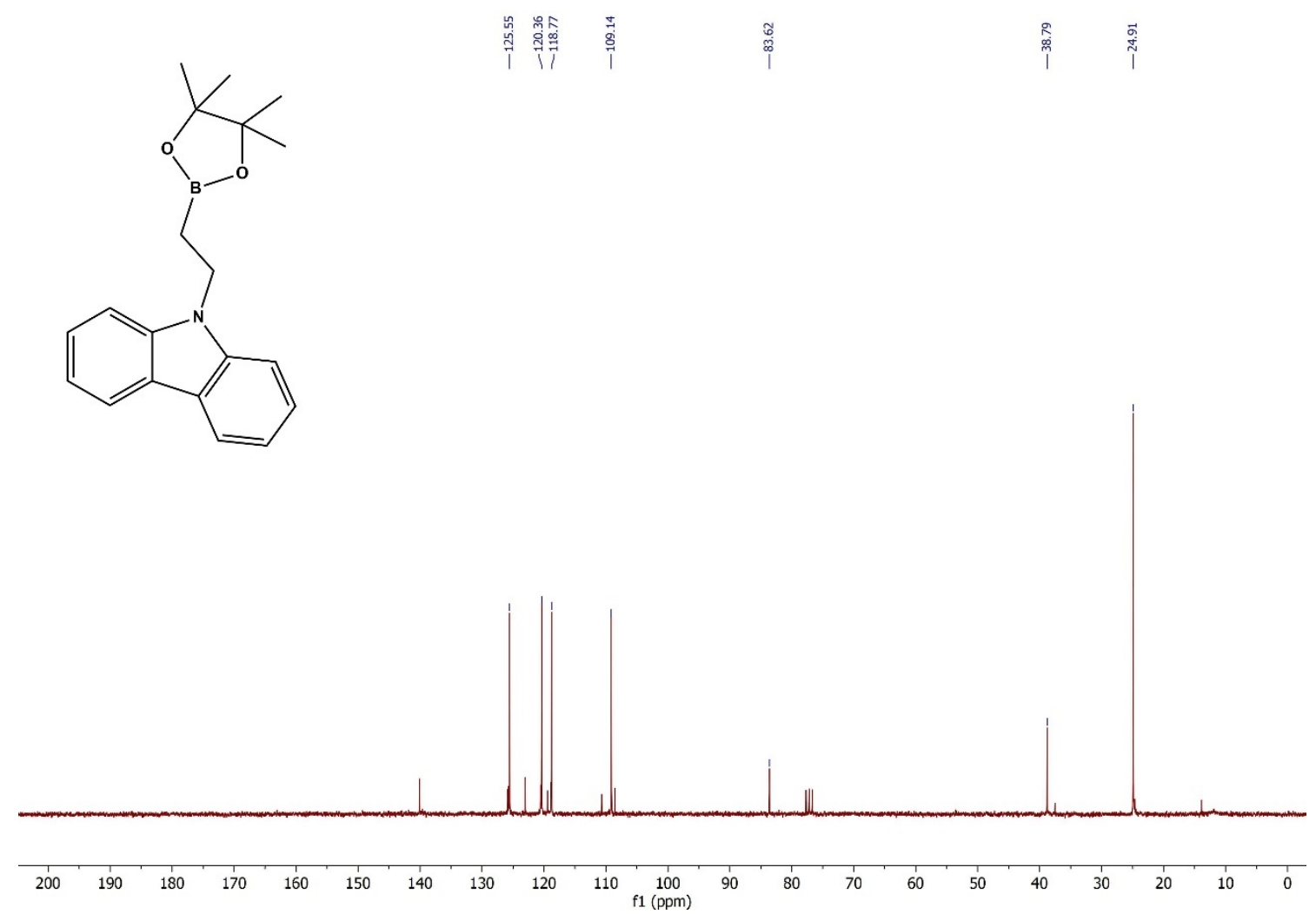

Figure S36. ${ }^{13} \mathrm{C}$ NMR Spectrum of 9-(2-(4,4,5,5-tetramethyl-1,3,2-dioxaborolan-2-yl)ethyl)-9Hcarbazole. 

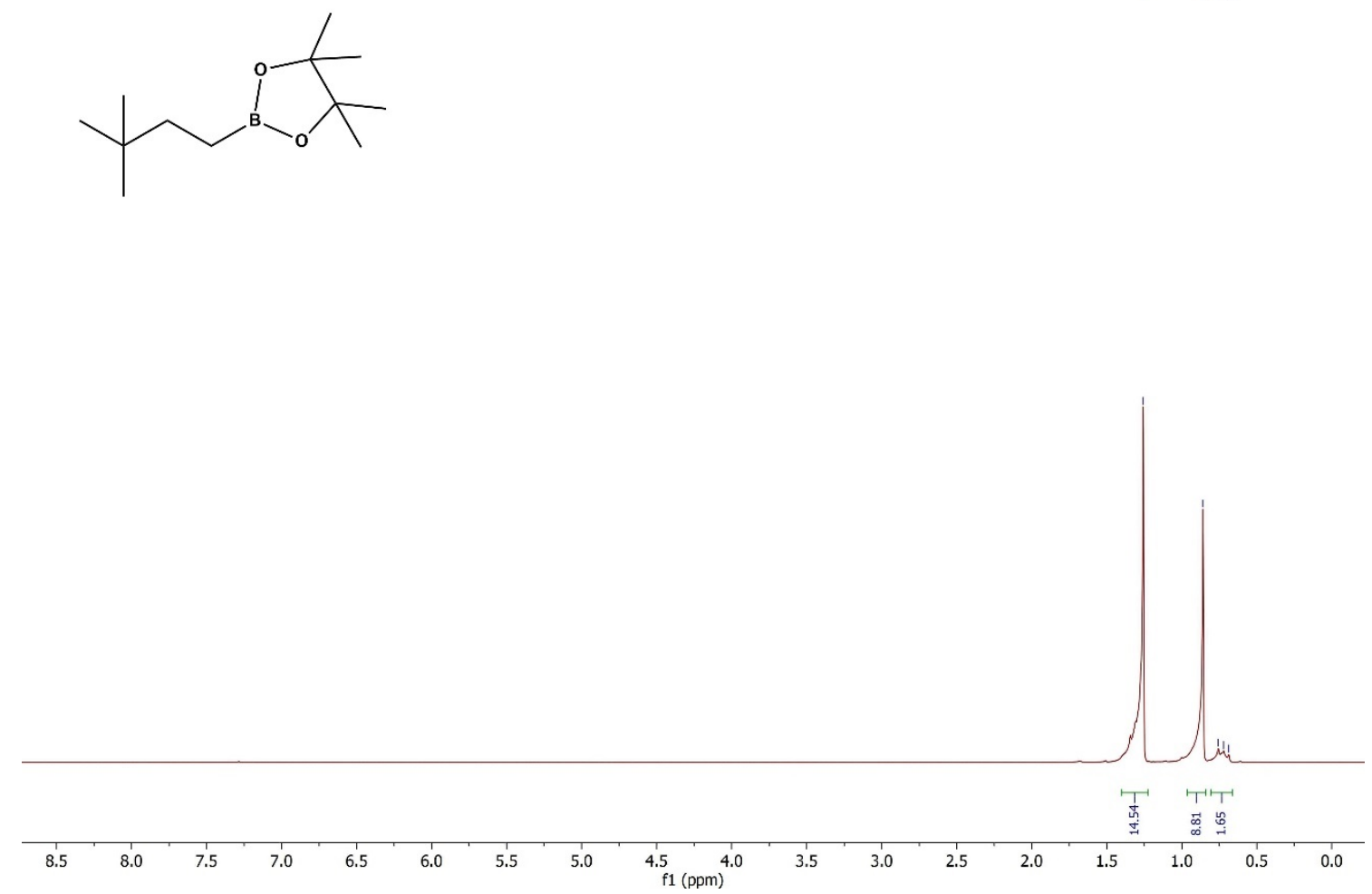

Figure S37. ${ }^{1} \mathrm{H}$ NMR Spectrum of 2-(3,3-dimethylbutyl)-4,4,5,5-tetramethyl-1,3,2-dioxaborolane.
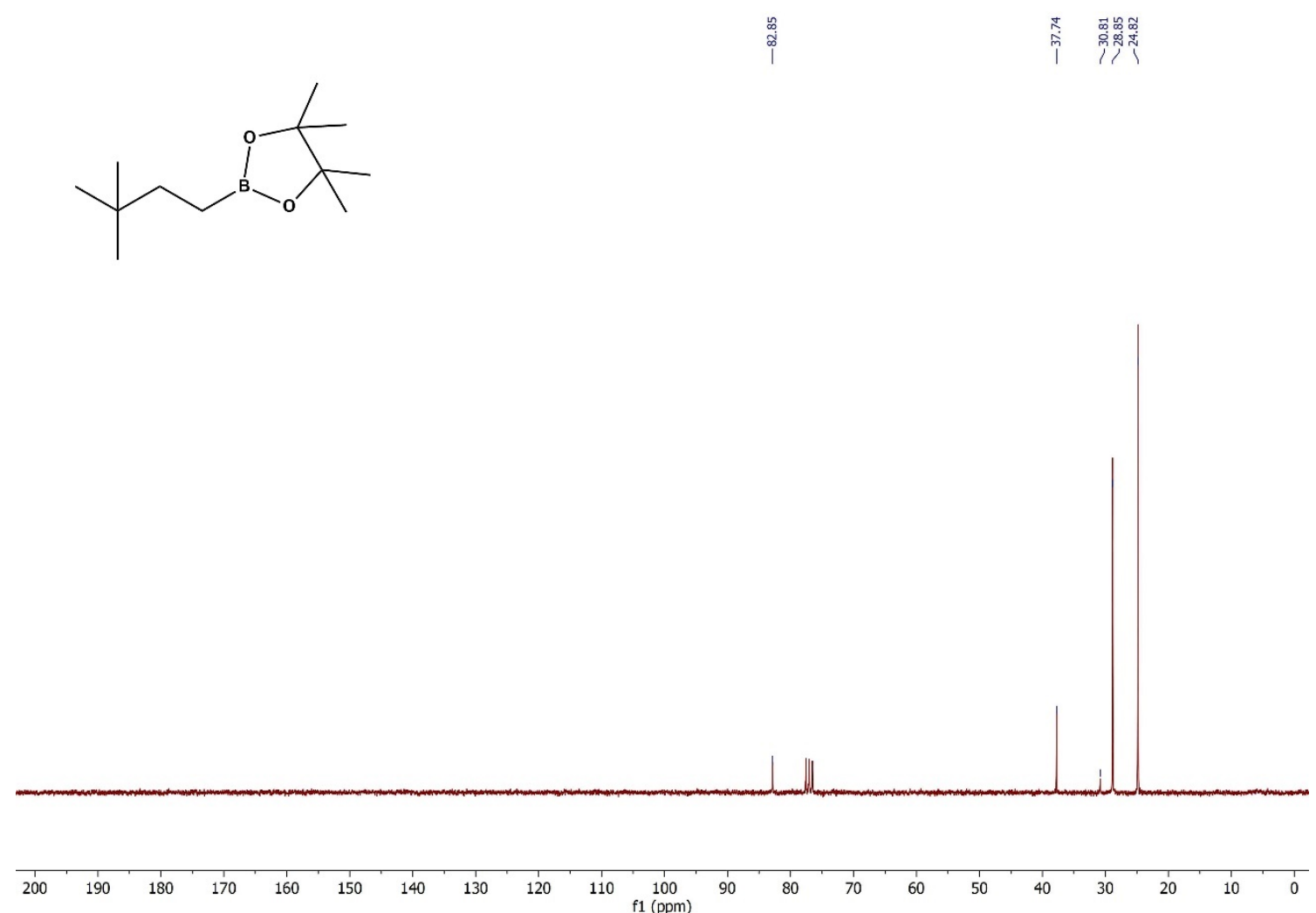

Figure S38. ${ }^{13} \mathrm{C}$ NMR Spectrum of 2-(3,3-dimethylbutyl)-4,4,5,5-tetramethyl-1,3,2-dioxaborolane. 


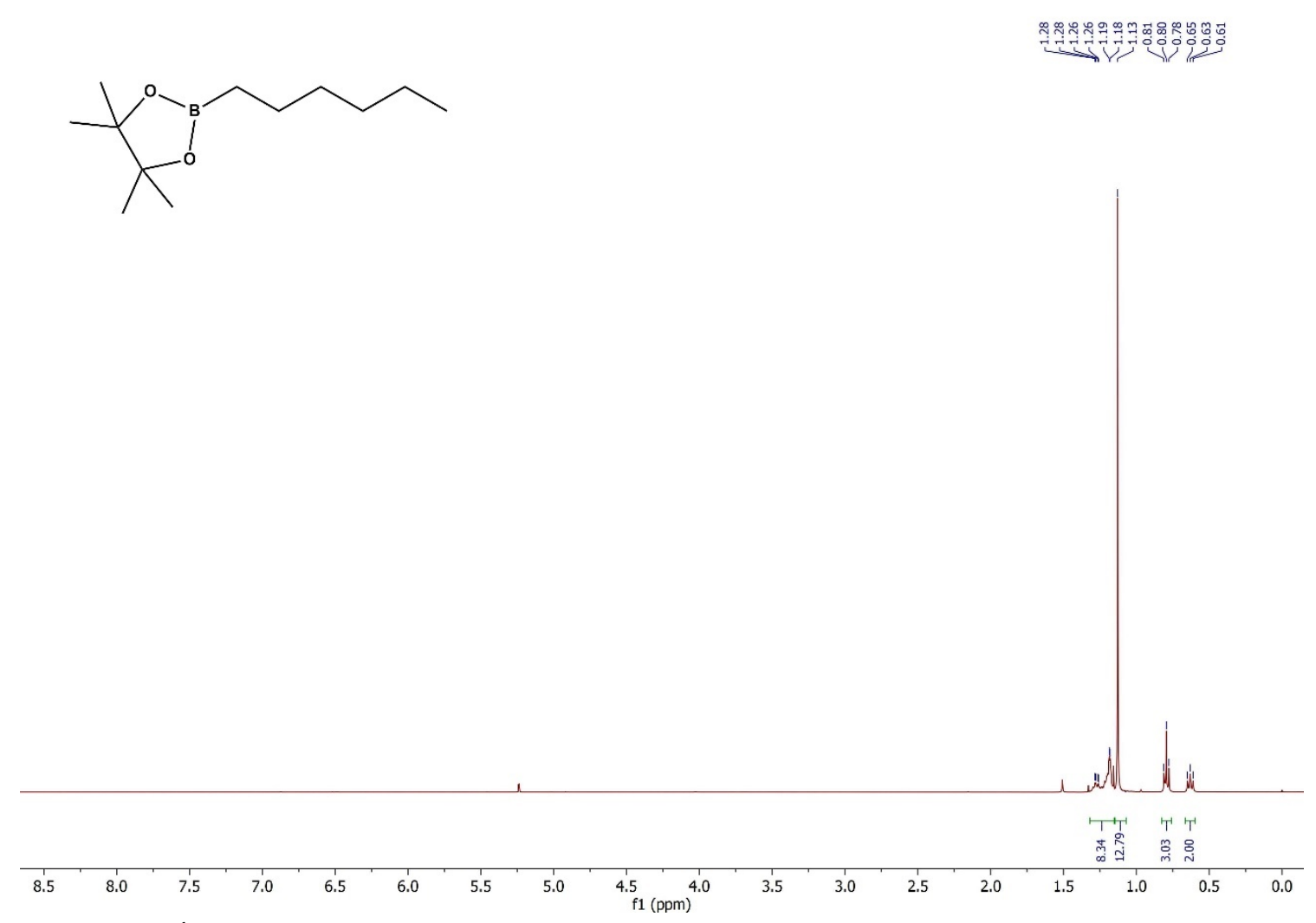

Figure S39. ${ }^{1} \mathrm{H}$ NMR Spectrum of 2-hexyl-4,4,5,5-tetramethyl-1,3,2-dioxaborolane.

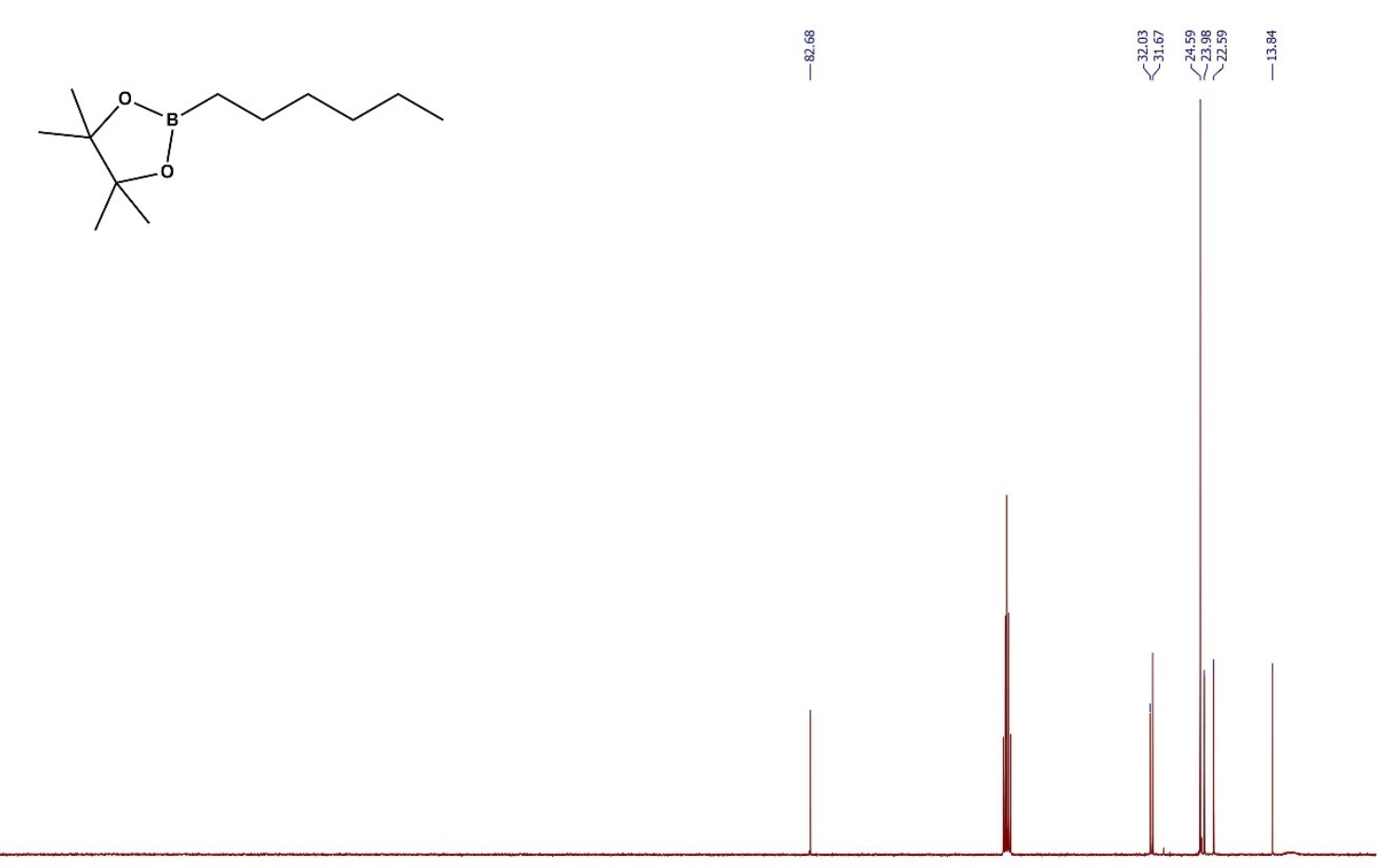

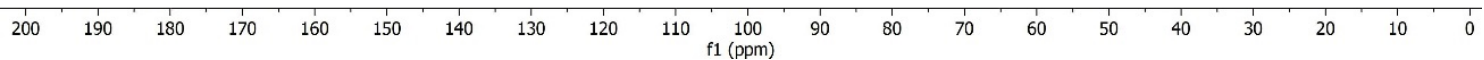

Figure S40. ${ }^{13} \mathrm{C}$ NMR Spectrum of 2-hexyl-4,4,5,5-tetramethyl-1,3,2-dioxaborolane. 


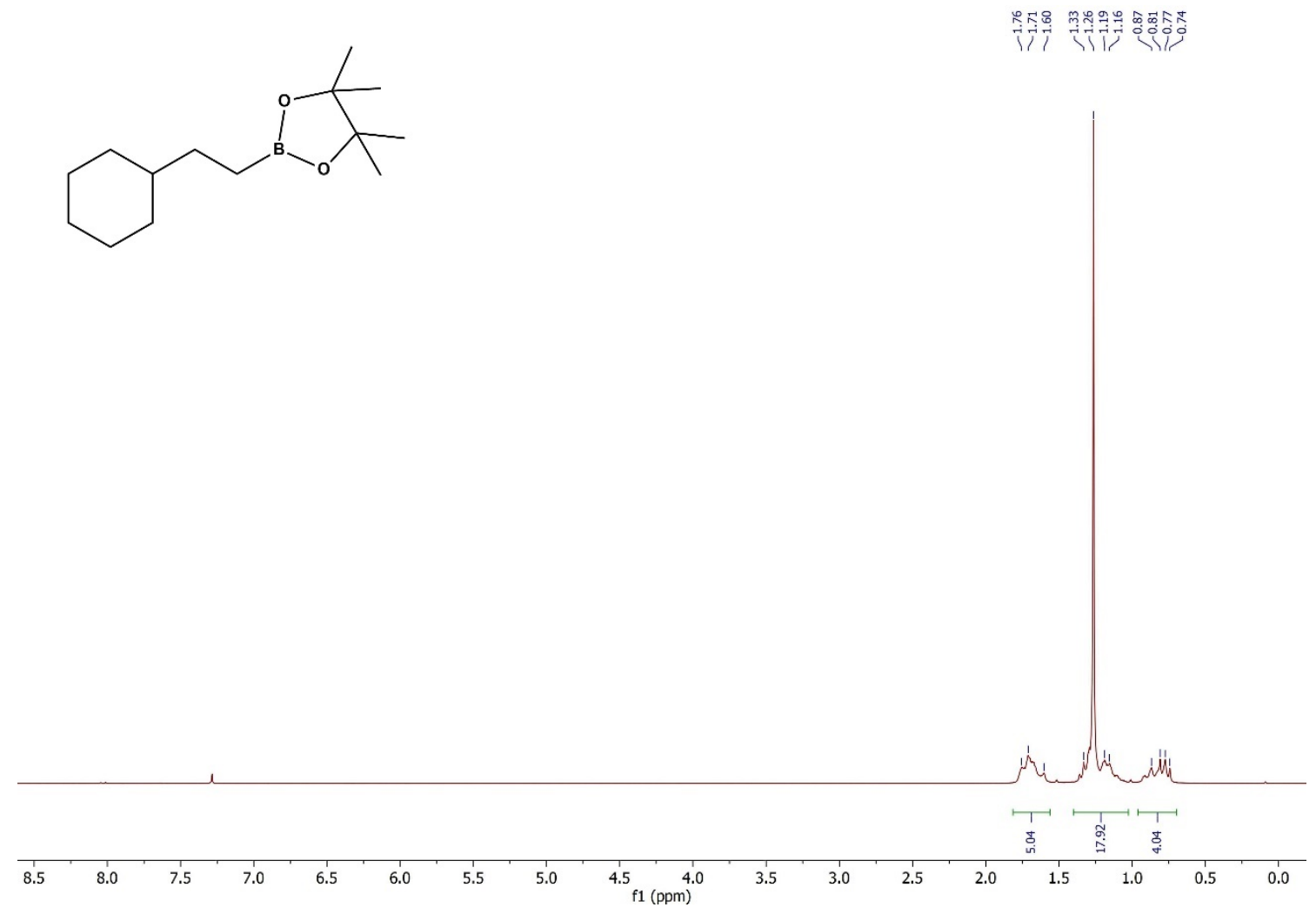

Figure S41. ${ }^{1} \mathrm{H}$ NMR Spectrum of 2-(2-cyclohexylethyl)-4,4,5,5-tetramethyl-1,3,2-dioxaborolane.

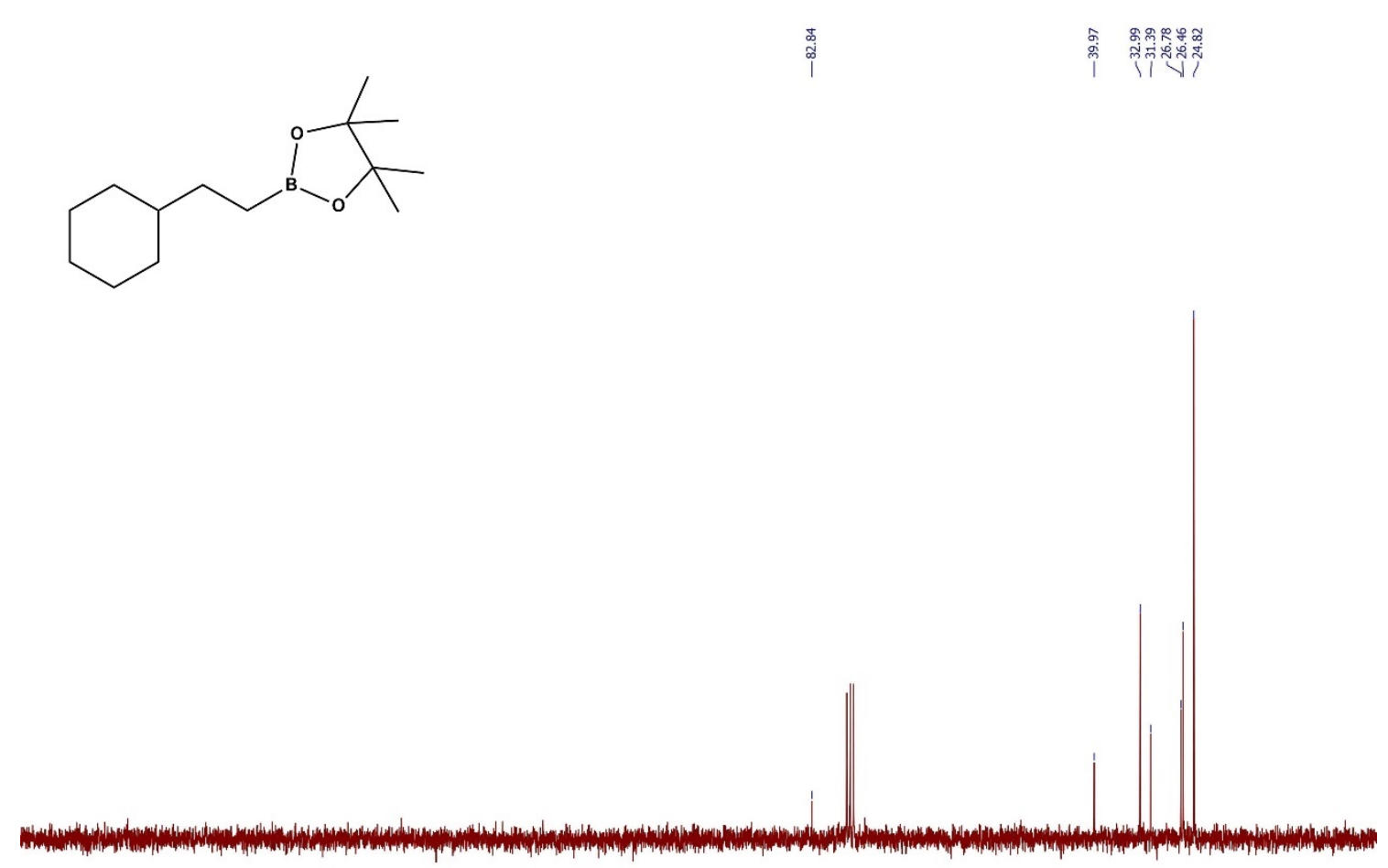

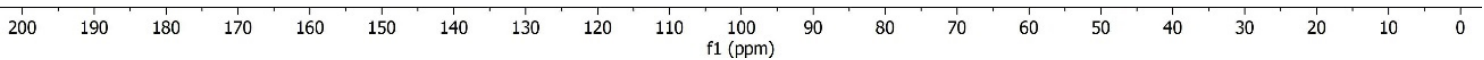

Figure S42. ${ }^{13} \mathrm{C}$ NMR Spectrum of 2-(2-cyclohexylethyl)-4,4,5,5-tetramethyl-1,3,2-dioxaborolane. 


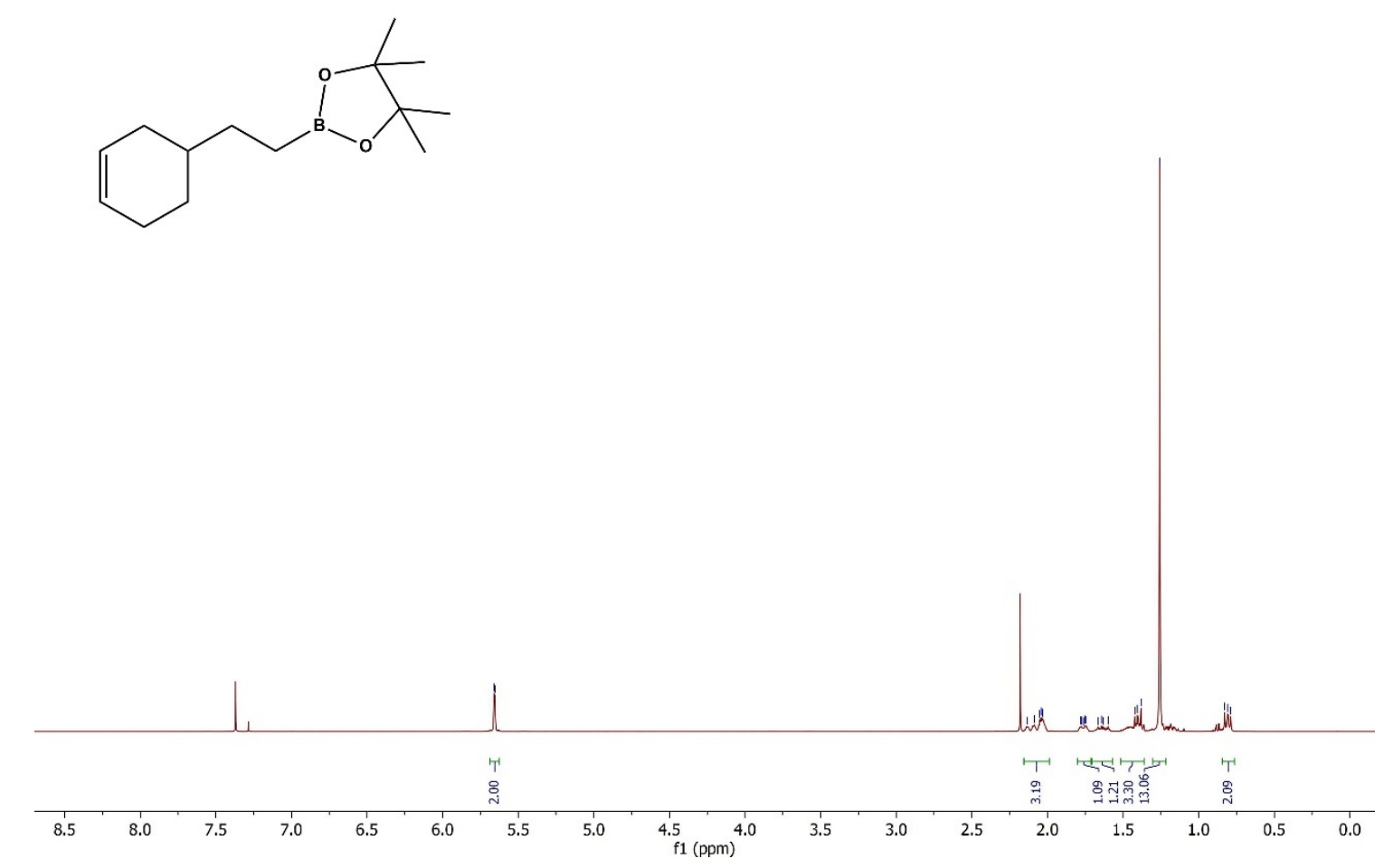

Figure S43. ${ }^{1} \mathrm{H}$ NMR Spectrum of 2-(2-(cyclohex-3-en-1-yl)ethyl)-4,4,5,5-tetramethyl-1,3,2dioxaborolane.
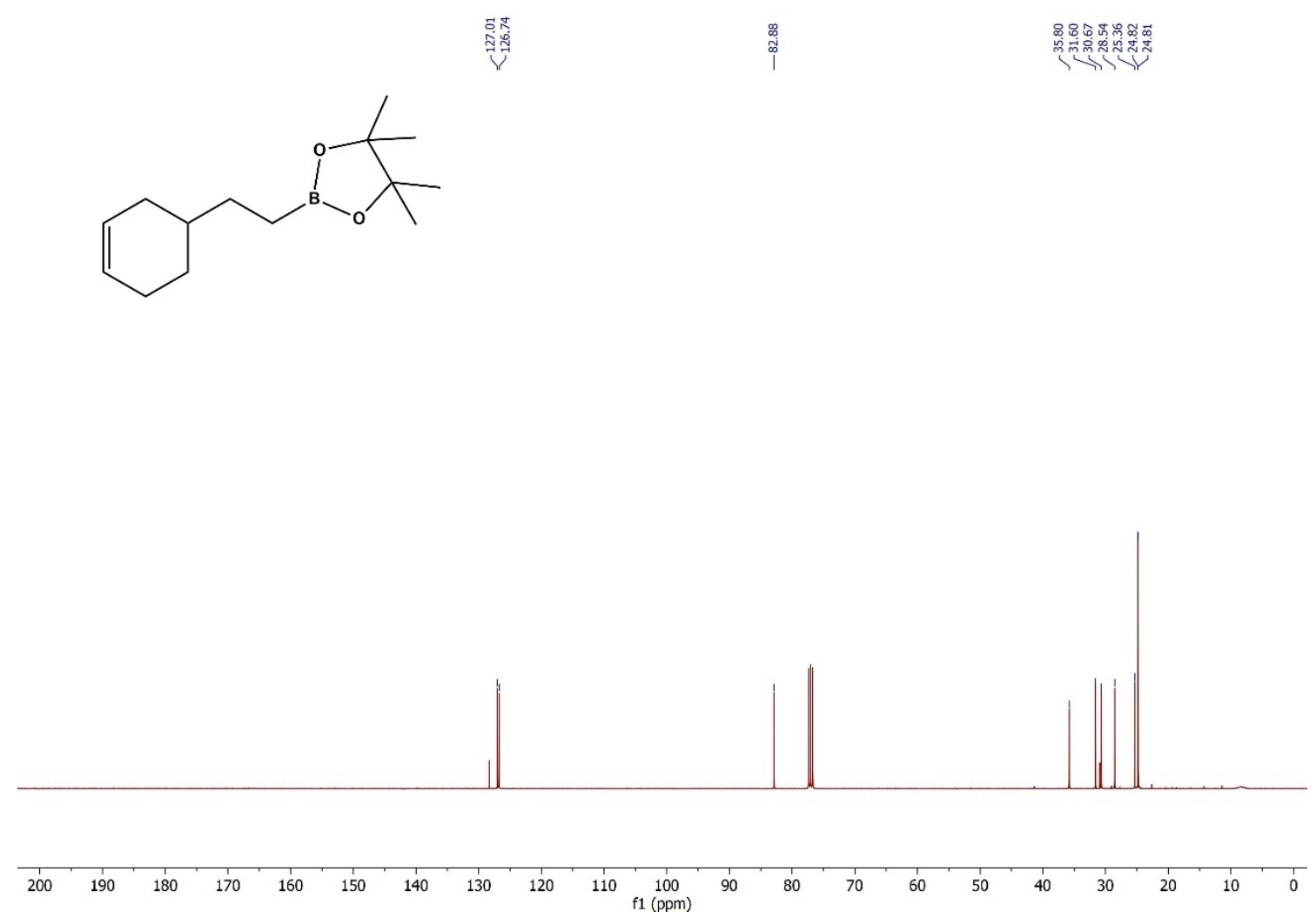

Figure S44. ${ }^{13} \mathrm{C}$ NMR Spectrum of 2-(2-(cyclohex-3-en-1-yl)ethyl)-4,4,5,5-tetramethyl-1,3,2dioxaborolane. 


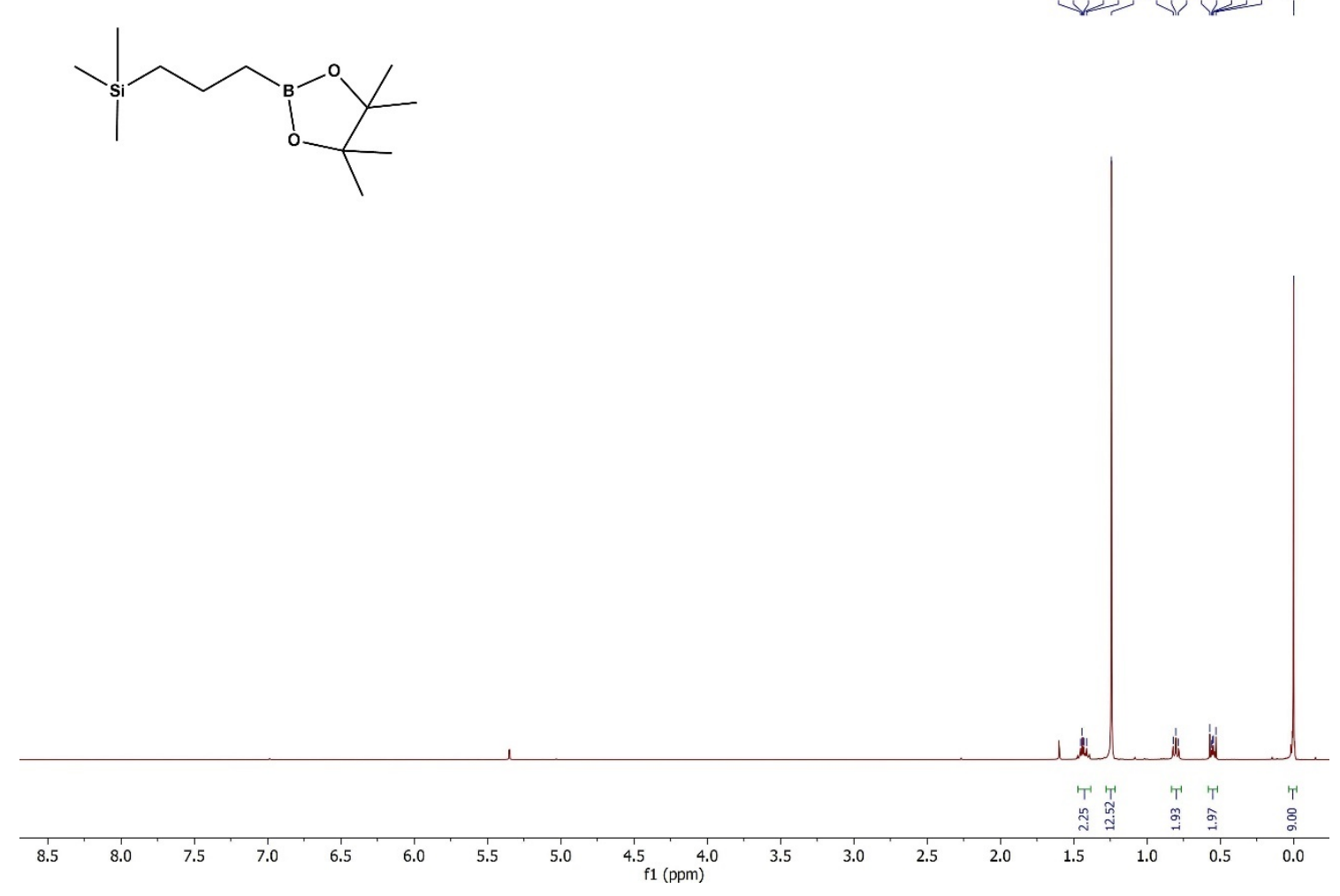

Figure S45. ${ }^{1} \mathrm{H}$ NMR Spectrum of Trimethyl(3-(4,4,5,5-tetramethyl-1,3,2-dioxaborolan-2yl)propyl)silane.

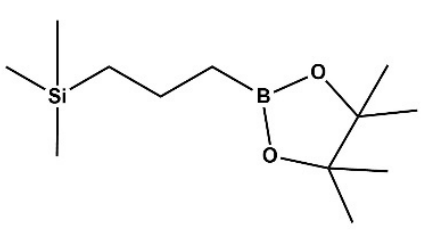

Figure S46. ${ }^{13} \mathrm{C}$ NMR Spectrum of Trimethyl(3-(4,4,5,5-tetramethyl-1,3,2-dioxaborolan-2yl)propyl)silane. 


\section{References}

[1] Kanas, D. A.; Geier, S. J.; Vogels, C. M.; Decken, A.; Westcott, S. A. Synthesis, Characterization, and Reactivity of Rhodium(I) Acetylacetonato Complexes Containing Pyridinecarboxaldimine Ligands. Inorg. Chem. 2008, 47, 8727-8735.

[2] Zhang, L.; Zuo, Z.; Lenge, X.; Huang, Z. A Cobalt-Catalyzed Alkene Hydroboration with Pinacolborane. Angew. Chem. Int. Ed. 2014, 53, 2696-2700.

[3] Cruz, T. F. C.; Pereira, L. C. J.; Waerenborgh, J. C.; Veiros, L. F.; Gomes, P. T. Hydroboration of terminal olefines with pinacolborane catalyzed by new 2-iminopyrrolyl iron(II) complexes. Catal. Sci. Technol. 2019, 9, 3347-3360.

[4] Liu, Y.; Zhou, Y.; Wang, H.; Qu, J. FeCl2-catalyzed hydroboration of aryl alkenes with bis(pinacolato)diboron. RSC Adv. 2015, 5, 73705-73713.

[5] Yamamoto, Y.; Fujikawa, R.; Umemoto, T.; Miyaura, N. Iridium-catalyzed hydroboration of alkenes with pinacolborane. Tetrahedron 2004, 60, 10695-10700.

[6] Garrett, C. E.; Fu, G. C. Hydroboration of Olefines with Catecholborane at Room Temperature in the Presence of N,N-Dimethylacetamide. J. Org. Chem. 1996, 61, 3224-3225. 